\title{
New model for radiatively generated Dirac neutrino masses and lepton flavor violating decays of the Higgs boson
}

\author{
Kazuki Enomoto, ${ }^{1, *}$ Shinya Kanemura, ${ }^{1, \dagger}$ Kodai Sakurai, ${ }^{2, \ddagger}$ and Hiroaki Sugiyama ${ }^{3,8}$ \\ ${ }^{1}$ Department of Physics, Osaka University, Toyonaka, Osaka 560-0043, Japan \\ ${ }^{2}$ Department of Physics, University of Toyama, 3190 Gofuku, Toyama 930-8555, Japan \\ ${ }^{3}$ Center for Liberal Arts and Sciences, Toyama Prefectural University, Toyama 939-0398, Japan
}

(Received 30 April 2019; published 29 July 2019)

\begin{abstract}
We propose a new mechanism to explain neutrino masses with lepton number conservation, in which the Dirac neutrino masses are generated at the two-loop level involving a dark matter candidate. In this model, branching ratios of lepton flavor violating decays of the Higgs boson can be much larger than those of lepton flavor violating decays of charged leptons. If lepton flavor violating decays of the Higgs boson are observed at future collider experiments without detecting lepton flavor violating decays of charged leptons, most of the models previously proposed for tiny neutrino masses are excluded while our model can still survive. We show that the model can be viable under constraints from current data for neutrino experiments, searches for lepton flavor violating decays of charged leptons, and dark matter experiments.
\end{abstract}

DOI: $10.1103 /$ PhysRevD.100.015044

\section{INTRODUCTION}

Although the Standard Model (SM) is consistent with the current data of collider experiments, there are still mysterious phenomena which cannot be explained in the SM, such as the origin of neutrino masses, the nature of dark matter and the baryon asymmetry of the Universe. To explain these phenomena by extending the SM is one of the central interests of today's high energy physics. Various models and mechanisms have also been proposed.

For the origin of neutrino masses, many new models have been studied along with the idea of the seesaw mechanism, which explains Majorana-type tiny neutrino masses by introducing new heavy particles, such as righthanded neutrinos [1,2], an additional isospin triplet scalar field [2,3] and isospin triplet fermions [4]. There is also an alternative scenario where tiny neutrino masses are generated by quantum effects. The first model along this line was proposed by Zee [5], in which one-loop effects

\footnotetext{
*kenomoto@het.phys.sci.osaka-u.ac.jp

${ }^{\dagger}$ kanemu@het.phys.sci.osaka-u.ac.jp

${ }^{\ddagger}$ Present address: Institute for Theoretical Physics, Karlsruhe Institute of Technology, D-76128 Karlsruhe, Germany; kodai .sakurai@kit.edu

$\S_{\text {shiro324@gmail.com }}$

Published by the American Physical Society under the terms of the Creative Commons Attribution 4.0 International license. Further distribution of this work must maintain attribution to the author(s) and the published article's title, journal citation, and DOI. Funded by SCOAP ${ }^{3}$.
}

due to an additional Higgs doublet field and a charged singlet scalar field yield Majorana-type tiny neutrino masses. There have been many variation models [612], some of which introduce an unbroken discrete symmetry in order not only to forbid tree-level generation of neutrino masses but also to guarantee the stability of extra particles in the loop so that the lightest one can be identified as a dark matter candidate [9-12]. In Ref. [11], an extended scalar sector for inducing neutrino masses at the three loop level with a dark matter candidate is also used to cause the strongly first order electroweak phase transition, which is required for successful electroweak baryogenesis [13].

In addition, models which generate Dirac-type tiny neutrino masses by quantum effects have also been proposed in Refs. [14-16]. In Ref. [16], introducing right-handed neutrinos with an odd quantum number under a new discrete symmetry, Dirac-type tiny neutrino masses are generated at the two-loop level. This model also has a dark matter candidate and can realize the strongly first order phase transition.

In Ref. [17], a class of models in which Majorana-type tiny neutrino masses are generated by quantum effects has been comprehensively studied by using flavor structures of induced neutrino mass matrices. Classification of models to generate Dirac-type neutrino masses has also been performed in Ref. [18].

Several years ago, anomaly for a lepton flavor violating (LFV) decay process of the Higgs boson $h \rightarrow \mu \tau$ at the LHC was reported by ATLAS [19] and CMS [20,21], although it disappeared soon later [22]. Motivated by this 
anomaly, the authors of Ref. [23] examined in a systematic way what kind of models for neutrino masses can predict a significant amount of signals for $h \rightarrow \mu \tau$. In Ref. [23], it was shown that most of the proposed models radiatively generating Majorana-type neutrino masses and Dirac-type neutrino masses, as well as minimal models of Type-I, II and III seesaw mechanisms are excluded if the signal of LFV decays of the Higgs boson is observed at future collider experiments without detecting LFV process for charged leptons. They also found that only a few models, in which Dirac-type neutrino masses are generated radiatively, may not be excluded even in this case.

In this paper, we concretely build one of such models, where additional scalar fields as well as right-handed fermions are introduced with even or odd charge under new discrete symmetries, so that Dirac-type tiny neutrino masses are generated at the two-loop level and a dark matter candidate is also contained. The branching ratio for LFV decays of the Higgs boson is not too small in spite of the stringent constraints from LFV processes for charged lepton decays. We will show that the model can be viable under the constraints from current data for neutrino experiments, searches for flavor violating decays of charged leptons and dark matter experiments.

This paper is organized as follows. In Sec. II, we define our model and introduce new fields and symmetries. In Sec. III, we give the formula of neutrino mass matrix which is generated at two-loop level. In Sec. IV, we consider the LFV processes $\ell \rightarrow \ell^{\prime} \gamma, h \rightarrow \ell \ell^{\prime}$ and $\ell_{m} \rightarrow \bar{\ell}_{n} \ell_{p} \ell_{q}$. In Sec. V, we show formulas of the thermally averaged cross sections of annihilation processes of the dark matter and the relic abundance. In Sec. VI, we present two benchmark scenarios and give numerical results of various phenomena in Secs. III-V. The first scenario is for the normal ordering mass hierarchy of neutrinos, and the second one is for the inverted ordering one. Conclusions are shown in Sec. VII. Some formulas are presented in Appendices.

\section{MODEL}

In our model, fields listed in Table I are added to the SM ones. We impose the conservation of the lepton number $L$ to our model. Gauge singlet right-handed fermions $\nu_{i R}$ $(i=1,2,3)$ have $L=1$, which compose three Dirac neutrinos with left-handed neutrinos $\nu_{\ell L}(\ell=e, \mu, \tau)$ of the SM lepton doublet fields $L_{\ell}=\left(\nu_{\ell L}, \ell_{L}\right)^{T}$. On the other hand, lepton numbers of the other gauge singlet fermions $\psi_{a R}(a=1,2,3)$ are zero. They have Majorana mass terms, $\frac{1}{2} M_{\psi_{a}} \overline{\psi_{a R}^{c}} \psi_{a R}$, without breaking the lepton number conservation, while Majorana mass terms of $\nu_{i R}$ are forbidden. If neutrinos have Yukawa interactions $\left(Y_{\nu}\right)_{\ell i} \overline{L_{\ell}} \phi^{c} \nu_{i R}$ with the SM Higgs doublet field $\phi=\left(\phi^{+}, \phi^{0}\right)^{T}$, masses of Dirac
TABLE I. The list of new fields in our model.

\begin{tabular}{lcccccc}
\hline \hline & $\nu_{i R}$ & $\psi_{a R}$ & $\Phi$ & $s_{1}^{+}$ & $\eta$ & $s_{2}^{+}$ \\
\hline Spin $J$ & $1 / 2$ & $1 / 2$ & 0 & 0 & 0 & 0 \\
$\operatorname{SU}(2)_{\mathrm{L}}$ & 1 & 1 & 2 & 1 & 2 & 1 \\
$\mathrm{U}(1)_{\mathrm{Y}}$ & 0 & 0 & $3 / 2$ & 1 & $1 / 2$ & 1 \\
$Z_{2}^{\prime}$ & - & + & $(+)$ & - & + & + \\
$L$ & 1 & 0 & -2 & -2 & -1 & -1 \\
$Z_{2}$ & + & - & + & + & - & - \\
\hline \hline
\end{tabular}

neutrinos can be generated with the vacuum expectation value $\left\langle\phi^{0}\right\rangle$. However, required values of Yukawa coupling constants $\left(Y_{\nu}\right)_{\ell i}$ for tiny neutrino masses seem to be unnaturally small. Thus, we impose a softly broken discrete symmetry $\left(Z_{2}^{\prime}\right)$ to our model in order to forbid tree-level Yukawa interaction of neutrinos, where $\nu_{i R}$ are odd under $Z_{2}^{\prime}$ while fields in the SM are even. Assignments of $Z_{2}^{\prime}$ quantum number to the new fields are shown in Table I. Although neutrino masses in the Lagrangian are forbidden by $Z_{2}^{\prime}$, they can be generated at the loop level via the soft breaking effect in the scalar sector. Throughout this paper, we take the basis where $\ell, \nu_{i R}$, and $\psi_{a R}$ are mass eigenstates.

Four new scalar fields $\left(\Phi, s_{1}^{+}, \eta\right.$, and $\left.s_{2}^{+}\right)$are involved in our model in addition to the Higgs doublet field $\phi$ of the SM. Both of $s_{1}^{+}$with $L=-2$ and $s_{2}^{+}$with $L=-1$ are $\mathrm{SU}(2)_{L}$-singlet fields with $Y=1$. On the other hand, $\Phi=$ $\left(\Phi^{++}, \Phi^{+}\right)^{T}$ with $Y=3 / 2$ and $\eta=\left(\eta^{+}, \eta^{0}\right)^{T}$ with $Y=$ $1 / 2$ are $\mathrm{SU}(2)_{L}$-doublet fields. The doublet field $\Phi$ has $L=-2$, and the even parity under $Z_{2}^{\prime}$ is assigned to $\Phi .{ }^{1}$ Although $\eta$ belongs to the same representation as $\phi$ under the SM gauge symmetry, $\eta$ has $L=-1$ in contrast with $L=0$ for $\phi$. We restrict ourselves to the case where $\eta^{0}$, the neutral component of $\eta$, does not have a vacuum expectation value in order to keep the lepton number conservation. The other new scalar fields do not also have vacuum expectation values because they are electrically charged.

Apart from $Z_{2}^{\prime}$, an accidental unbroken discrete symmetry $\left(Z_{2}\right)$ appears in our model due to the lepton number conservation, Majorana mass terms of $\psi_{a R}$ and some of new Yukawa interactions, ${ }^{2}$ where the parity is given by $(-1)^{L+2 J}$. Three fields $\left(\psi_{a R}, \eta\right.$, and $\left.s_{2}^{+}\right)$are odd under $Z_{2}$. The lightest $Z_{2}$-odd particle is stable. If $\psi_{a R}$ or $\eta^{0}$ is the lightest one, it can be a dark matter candidate.

In our model, there are three new Yukawa interactions as

$$
\begin{aligned}
\mathcal{L}_{\text {Yukawa }}= & \left(Y_{1}\right)_{\ell i} \overline{\left(\ell_{R}\right)^{c}} \nu_{i R} s_{1}^{+}+\left(Y_{2}\right)_{\ell a} \overline{\left(\ell_{R}\right)^{c}} \psi_{a R} s_{2}^{+} \\
& +\left(Y_{\eta}\right)_{\ell a} \overline{L_{\ell}} \eta^{c} \psi_{a R}+\text { H.c. }
\end{aligned}
$$

\footnotetext{
${ }^{1}$ Actually, the $Z_{2}^{\prime}$ parity of $\Phi$ is irrelevant to our study in this article so that the odd-parity is also acceptable for $\Phi$.

${ }^{2}$ These Majorana mass terms, $Y_{1}$ and $Y_{2}$ terms in Eq. (1) explicitly break $\mathrm{U}(1)_{L+2 J}$ into its $Z_{2}$ subgroup.
} 
The scalar potential is given by

$$
\begin{aligned}
V= & \mu_{1}^{2}|\phi|^{2}+\mu_{2}^{2}|\Phi|^{2}+\mu_{3}^{2}\left|s_{1}^{+}\right|^{2}+\mu_{4}^{2}|\eta|^{2}+\mu_{5}^{2}\left|s_{2}^{+}\right|^{2} \\
& +\left(\sigma_{1} \Phi^{\dagger} \phi s_{1}^{+}+\text {H.c. }\right)+\left(\sigma_{2} \Phi^{\dagger} \eta s_{2}^{+}+\text {H.c. }\right)+\left(\sigma_{3} \phi^{\dagger} \eta^{c} s_{2}^{+}+\text {H.c. }\right) \\
& +\lambda_{\phi}|\phi|^{4}+\lambda_{\Phi}|\Phi|^{4}+\lambda_{1}\left|s_{1}^{+}\right|^{4}+\lambda_{\eta}|\eta|^{4}+\lambda_{2}\left|s_{2}^{+}\right|^{4} \\
& +\lambda_{\phi \Phi}|\phi|^{2}|\Phi|^{2}+\lambda_{\phi \Phi}^{\prime}\left|\phi^{\dagger} \Phi\right|^{2}+\lambda_{\phi \eta}|\phi|^{2}|\eta|^{2}+\lambda_{\phi \eta}^{\prime}\left|\phi^{\dagger} \eta\right|^{2}+\lambda_{\Phi \eta}|\Phi|^{2}|\eta|^{2}+\lambda_{\Phi \eta}^{\prime}\left|\Phi^{\dagger} \eta\right|^{2} \\
& +\sum_{k=1}^{2}\left\{\lambda_{\phi k}|\phi|^{2}\left|s_{k}\right|^{2}+\lambda_{\Phi k}|\Phi|^{2}\left|s_{k}\right|^{2}+\lambda_{\eta k}|\eta|^{2}\left|s_{k}\right|^{2}\right\}+\lambda_{12}\left|s_{1}^{+}\right|^{2}\left|s_{2}^{+}\right|^{2} \\
& +\left(\xi_{1} \eta^{\dagger} \Phi \eta^{\dagger} \phi^{c}+\text { H.c. }\right)+\left(\xi_{2} \Phi^{\dagger} \phi^{c}\left(s_{2}^{+}\right)^{2}+\text { H.c. }\right) .
\end{aligned}
$$

Notice that $\sigma_{1}$ is the soft breaking parameter for $Z_{2}^{\prime}{ }^{3}$ There are five complex coupling constants $\left(\sigma_{1}, \sigma_{2}, \sigma_{3}, \xi_{1}\right.$, and $\left.\xi_{2}\right)$, and two $C P$-violating phases remain as physical parameters after redefinitions of phases of fields. ${ }^{4}$ In this article, coupling constants in the scalar potential are taken to be real, just for simplicity.

The SM Higgs doublet field $\phi$ does not mix with the other scalar fields in our model. Thus, identically to the $\mathrm{SM}$, the field can be expressed as $\phi=\left(G^{+},\left(v+h+i G^{0}\right) /\right.$ $\sqrt{2})^{T}$, where $v\left(=\sqrt{-\mu_{1}^{2} / \lambda_{\phi}}=246 \mathrm{GeV}\right)$ is the vacuum expectation value. The real component $h$ corresponds to the SM Higgs boson, whose mass is given by $m_{h}=\sqrt{2 \lambda_{\phi}} v$. Nambu-Goldston bosons $\left(G^{+}\right.$and $\left.G^{0}\right)$ are absorbed by the longitudinally polarized weak gauge bosons by the electroweak symmetry breaking.

Fields $\Phi^{++}$and $\eta^{0}$ are mass eigenstates. Their squared masses are given by

$$
\begin{gathered}
m_{\Phi^{++}}^{2}=\mu_{2}^{2}+\frac{1}{2} \lambda_{\phi \Phi} v^{2}, \\
m_{\eta^{0}}^{2}=\mu_{4}^{2}+\frac{1}{2}\left(\lambda_{\phi \eta}+\lambda_{\eta \phi}^{\prime}\right) v^{2} .
\end{gathered}
$$

Mass eigenstates $\pi_{1}^{+}$and $\pi_{2}^{+}$, which are singly charged and have $L=-2$, are obtained by linear combinations of $\Phi^{+}$ and $s_{1}^{+}$as

$$
\left(\begin{array}{c}
\pi_{1}^{+} \\
\pi_{2}^{+}
\end{array}\right)=U_{\theta}\left(\begin{array}{c}
\Phi^{+} \\
s_{1}^{+}
\end{array}\right), \quad U_{\theta}=\left(\begin{array}{cc}
\cos \theta & \sin \theta \\
-\sin \theta & \cos \theta
\end{array}\right)
$$

where the mixing angle $\theta$ is defined as

\footnotetext{
${ }^{3}$ If $\Phi$ is taken to be odd under $Z_{2}^{\prime}$, the soft breaking parameter is $\sigma_{2}$. Therefore, a product $\sigma_{1} \sigma_{2}$ breaks $Z_{2}^{\prime}$ independently of the $Z_{2}^{\prime}$ parity of $\Phi$.

${ }^{4}$ If we take $\Phi$ as a $Z_{2}^{\prime}$-odd field, terms of $\xi_{1}$ and $\xi_{2}$ are replaced with $\xi_{3} \Phi^{\dagger} \eta^{c} s_{1}^{+} s_{2}^{+}$. Then, only one $C P$-violating phase is physical.
}

$$
\begin{aligned}
\tan 2 \theta & =\frac{-2\left(M_{\Phi S_{1}}^{2}\right)_{12}}{\left(M_{\Phi S_{1}}^{2}\right)_{22}-\left(M_{\Phi S_{1}}^{2}\right)_{11}}, \\
M_{\Phi S_{1}}^{2} & =\left(\begin{array}{cc}
\mu_{2}^{2}+\frac{1}{2}\left(\lambda_{\phi \Phi}+\lambda_{\phi \Phi}^{\prime}\right) v^{2} & \frac{1}{\sqrt{2}} \sigma_{1} v \\
\frac{1}{\sqrt{2}} \sigma_{1} v & \mu_{3}^{2}+\frac{1}{2} \lambda_{\phi 1} v^{2}
\end{array}\right) .
\end{aligned}
$$

Squared masses of $\pi_{1}^{+}$and $\pi_{2}^{+}$are given by

$$
\begin{aligned}
m_{\pi_{1}}^{2}= & \frac{1}{2}\left\{\left(M_{\Phi S_{1}}^{2}\right)_{11}+\left(M_{\Phi s_{1}}^{2}\right)_{22}\right. \\
& \left.+\sqrt{\left(\left(M_{\Phi S_{1}}^{2}\right)_{22}-\left(M_{\Phi S_{1}}^{2}\right)_{11}\right)^{2}+4\left(M_{\Phi S_{1}}^{2}\right)_{12}^{2}}\right\}, \\
m_{\pi_{2}}^{2}= & \frac{1}{2}\left\{\left(M_{\Phi S_{1}}^{2}\right)_{11}+\left(M_{\Phi S_{1}}^{2}\right)_{22}\right. \\
& \left.-\sqrt{\left(\left(M_{\Phi S_{1}}^{2}\right)_{22}-\left(M_{\Phi S_{1}}^{2}\right)_{11}\right)^{2}+4\left(M_{\Phi S_{1}}^{2}\right)_{12}^{2}}\right\} .
\end{aligned}
$$

Mass eigenstates $\omega_{1}^{+}$and $\omega_{2}^{+}$, which are $Z_{2}$-odd with $L=-1$, are constructed by linear combinations of $\eta^{+}$and $s_{2}^{+}$as follows:

$$
\left(\begin{array}{c}
\omega_{1}^{+} \\
\omega_{2}^{+}
\end{array}\right)=U_{\chi}\left(\begin{array}{c}
\eta^{+} \\
s_{2}^{+}
\end{array}\right), \quad U_{\chi}=\left(\begin{array}{cc}
\cos \chi & \sin \chi \\
-\sin \chi & \cos \chi
\end{array}\right),
$$

where the mixing angle $\chi$ is defined as

$$
\begin{aligned}
\tan 2 \chi & =\frac{-2\left(M_{\eta s_{2}}^{2}\right)_{12}}{\left(M_{\eta s_{2}}^{2}\right)_{22}-\left(M_{\eta s_{2}}^{2}\right)_{11}}, \\
M_{\eta s_{2}}^{2} & =\left(\begin{array}{cc}
\mu_{4}^{2}+\frac{1}{2} \lambda_{\phi \eta} v^{2} & -\frac{1}{\sqrt{2}} \sigma_{3} v \\
-\frac{1}{\sqrt{2}} \sigma_{3} v & \mu_{5}^{2}+\frac{1}{2} \lambda_{\phi 2} v^{2}
\end{array}\right) .
\end{aligned}
$$

Squared masses of $\omega_{1}^{+}$and $\omega_{2}^{+}$are given by

$$
\begin{aligned}
m_{\omega_{1}}^{2}= & \frac{1}{2}\left\{\left(M_{\eta s_{2}}^{2}\right)_{11}+\left(M_{\eta s_{2}}^{2}\right)_{22}\right. \\
& \left.+\sqrt{\left(\left(M_{\eta s_{2}}^{2}\right)_{22}-\left(M_{\eta s_{2}}^{2}\right)_{11}\right)^{2}+4\left(M_{\eta s_{2}}^{2}\right)_{12}^{2}}\right\},
\end{aligned}
$$




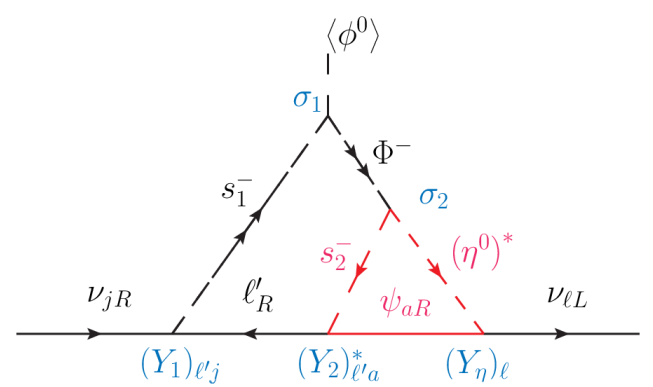

FIG. 1. The Feynman diagram to generate Dirac-type neutrino masses. Arrows denote flows of the conserved lepton number. Red colored lines represent those of $Z_{2}$-odd fields.

$$
\begin{aligned}
m_{\omega_{2}}^{2}= & \frac{1}{2}\left\{\left(M_{\eta s_{2}}^{2}\right)_{11}+\left(M_{\eta s_{2}}^{2}\right)_{22}\right. \\
& \left.-\sqrt{\left(\left(M_{\eta s_{2}}^{2}\right)_{22}-\left(M_{\eta s_{2}}^{2}\right)_{11}\right)^{2}+4\left(M_{\eta s_{2}}^{2}\right)_{12}^{2}}\right\} .
\end{aligned}
$$

\section{NEUTRINO MASS}

Mass terms $\left(m_{D}\right)_{\ell i} \nu_{\ell L}^{-} \nu_{i R}$ of Dirac neutrinos are generated in our model via two-loop diagrams in Fig. 1. The Dirac neutrino mass matrix $\left(m_{D}\right)_{\ell i}$ is calculated as

$$
\begin{aligned}
\left(m_{\nu}\right)_{\ell i}= & \frac{\left(m_{\pi_{2}}^{2}-m_{\pi_{1}}^{2}\right) \sigma_{2} \sin (2 \theta)}{2} \\
& \times \sum_{\ell^{\prime}, a, k}\left(Y_{\eta}\right)_{\ell a}\left(Y_{2}\right)_{\ell^{\prime} a}^{*}\left(Y_{1}\right)_{\ell^{\prime} i}\left(U_{\chi}\right)_{k 2}^{2} I_{\ell^{\prime} a k},
\end{aligned}
$$

where the coupling constant $\sigma_{1}$ in Fig. 1 is replaced by using $2 \sigma_{1}\left\langle\phi^{0}\right\rangle=\left(m_{\pi_{1}}^{2}-m_{\pi_{2}}^{2}\right) \sin (2 \theta)$. The explicit formula for the loop function $I_{\ell^{\prime} a k}$ is given in Appendix A. Notice that $\sigma_{2} \sin (2 \theta)$ softly breaks $Z_{2}^{\prime}$ that forbids $\overline{L_{\ell}} \phi^{c} \nu_{i R}$.

Since we take the basis where $\nu_{i R}$ are mass eigenstates, the neutrino mass matrix $\left(m_{\nu}\right)_{\ell i}$ is diagonalized as

$$
m_{\nu}=U_{\mathrm{PMNS}} \operatorname{diag}\left(m_{1}, m_{2}, m_{3}\right),
$$

where $m_{i}(i=1,2,3)$ denote masses of Dirac neutrinos. The mixing matrix $U_{\mathrm{PMNS}}$ is the Pontecorvo-MakiNakagawa-Sakata matrix $[24,25]$, which can be parametrized as

$$
\begin{aligned}
U_{\mathrm{PMNS}}= & \left(\begin{array}{ccc}
1 & 0 & 0 \\
0 & c_{23} & s_{23} \\
0 & -s_{23} & c_{23}
\end{array}\right)\left(\begin{array}{ccc}
c_{13} & 0 & s_{13} e^{-i \delta} \\
0 & 1 & 0 \\
-s_{13} e^{i \delta} & 0 & c_{13}
\end{array}\right) \\
& \times\left(\begin{array}{ccc}
c_{12} & s_{12} & 0 \\
-s_{12} & c_{12} & 0 \\
0 & 0 & 1
\end{array}\right),
\end{aligned}
$$

where $c_{i j}=\cos \theta_{i j}$ and $s_{i j}=\sin \theta_{i j}$, and $\delta$ is a $C P$ violating phase in the lepton sector.

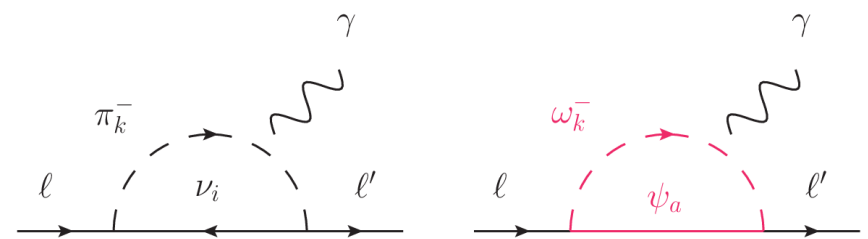

FIG. 2. Feynman diagrams for charged lepton LFV processes $\ell \rightarrow \ell^{\prime} \gamma$

\section{LEPTON FLAVOR VIOLATION}

Matrices $Y_{1}, Y_{2}$, and $Y_{\eta}$ are not diagonal and cause LFV processes. Radiative decays of charged leptons, $\ell \rightarrow \ell^{\prime} \gamma$, can be caused via the one-loop diagrams in Fig. 2. Ignoring $m_{\ell^{\prime}}$, branching ratios of these decays are expressed as

$\frac{\operatorname{Br}\left(\ell \rightarrow \ell^{\prime} \gamma\right)}{\operatorname{Br}\left(\ell \rightarrow \ell^{\prime} \nu_{\ell} \overline{\nu_{\ell^{\prime}}}\right)}=\frac{3}{16 \pi} \frac{\alpha}{G_{F}^{2} m_{\ell}^{4}}\left(\left|A_{R}^{s_{1}}+A_{R}^{\omega}\right|^{2}+\left|A_{L}^{\omega}\right|^{2}\right)$,

where $\alpha$ is the fine-structure constant, $G_{F}=1.17 \times$ $10^{-5} \mathrm{GeV}^{-2}$ is the Fermi constant, $\operatorname{Br}\left(\tau \rightarrow e \nu_{\tau} \overline{\nu_{e}}\right) \simeq$ 0.178, $\operatorname{Br}\left(\tau \rightarrow \mu \nu_{\tau} \overline{\nu_{\mu}}\right) \simeq 0.174$, and $\operatorname{Br}\left(\mu \rightarrow e \nu_{\mu} \overline{\nu_{e}}\right) \simeq 1$ [26]. Formulas of $A_{R}^{s_{1}}, A_{R}^{\omega}$, and $A_{L}^{\omega}$ are presented in Appendix B. $A_{R}^{s_{1}}$ corresponds to the contribution from $s_{1}^{+}$to $\ell \rightarrow \ell_{R}^{\prime} \gamma$. Contributions of $s_{2}^{+}$and $\eta^{+}$to $\ell \rightarrow \ell_{R}^{\prime} \gamma$ are given by $A_{R}^{\omega}$, while $A_{L}^{\omega}$ is for their contributions to $\ell \rightarrow \ell_{L}^{\prime} \gamma$.

Scalar fields that contribute to $\ell \rightarrow \ell^{\prime} \gamma$ affect also $h \rightarrow \ell \ell^{\prime}\left(\ell \neq \ell^{\prime}\right)$ via diagrams in Fig. 3. Decay widths for $h \rightarrow \ell \ell^{\prime}\left(\ell \neq \ell^{\prime}\right)$ are given by

$$
\begin{aligned}
\Gamma\left(h \rightarrow \ell \ell^{\prime}\right) & =\Gamma\left(h \rightarrow \bar{\ell} \ell^{\prime}\right)+\Gamma\left(h \rightarrow \bar{\ell}^{\prime} \ell\right) \\
& =\frac{m_{h}}{8 \pi}\left(\frac{1}{16 \pi^{2}}\right)^{2}\left(\left|B_{R}^{s_{1}}+B_{R}^{\omega}\right|^{2}+\left|B_{L}^{\omega}\right|^{2}\right),
\end{aligned}
$$

where we take $m_{\ell^{\prime}}=0$. Formulas of $B_{R}^{s_{1}}, B_{R}^{\omega}$, and $B_{L}^{\omega}$ are shown in Appendix $C$. The contribution from $s_{1}^{+}$is given by $B_{R}^{S_{1}}$, while those from $s_{2}^{+}$and $\eta^{+}$are involved in both of $B_{R}^{\omega}$ and $B_{L}^{\omega}$. The subscript $X(=L, R)$ in these $B_{X}$ 's indicates the chirality of the lighter charged lepton $\ell_{X}^{\prime}$ in the final state.

New scalar bosons in our model contribute also to $\ell_{m} \rightarrow$ $\bar{\ell}_{n} \ell_{p} \ell_{q}(m=2,3$ and $n, p, q=1,2)$ with new Yukawa interactions, where $\ell_{1}, \ell_{2}$ and $\ell_{3}$ corresponds to $e, \mu$, and $\tau$,

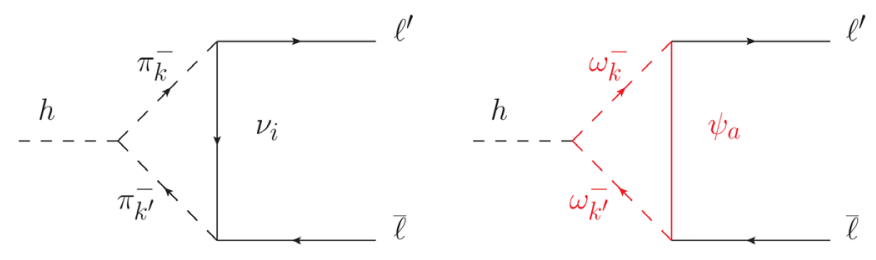

FIG. 3. Diagrams for $h \rightarrow \ell \bar{\ell}^{\prime}$. 


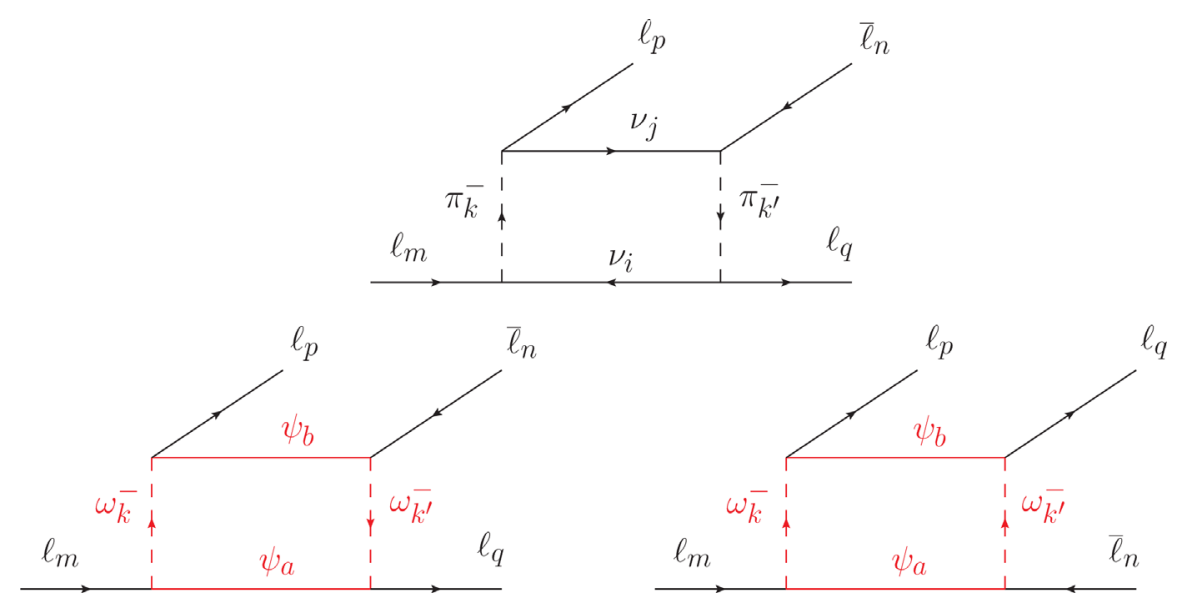

FIG. 4. Feynman diagrams for $\ell_{m} \rightarrow \bar{\ell}_{n} \ell_{p} \ell_{q}$.

respectively. Contributions from penguin diagrams can be ignored because of the constraint from $\ell \rightarrow \ell^{\prime} \gamma$. However, if some coupling constants of new Yukawa interactions are $O(1)$, box diagrams in Fig. 4 should be considered. Branching ratios for $\ell_{m} \rightarrow \bar{\ell}_{n} \ell_{p} \ell_{q}$ via the box diagrams are given by

$$
\begin{aligned}
& \frac{\operatorname{Br}\left(\ell_{m} \rightarrow \bar{\ell}_{n} \ell_{p} \ell_{q}\right)}{\operatorname{Br}\left(\ell_{m} \rightarrow \ell_{p} \nu_{\ell_{m}} \bar{\nu}_{\ell_{p}}\right)} \\
& =\frac{S}{64 G_{F}^{2}}\left(\frac{1}{16 \pi^{2}}\right)^{2}\left\{4 \left(\left|\left(C_{R R R R}^{s_{1}}\right)_{m n p q}+\left(C_{R R R R}^{s_{2}}\right)_{m n p q}\right|^{2}\right.\right. \\
& \left.\quad+\left|\left(C_{L L L L}^{\eta}\right)_{m n p q}\right|^{2}\right)+\left|\left(C_{L L R R}^{\omega}\right)_{m n p q}\right|^{2}+\left|\left(C_{L L R R}^{\omega}\right)_{m n q p}\right|^{2} \\
& \quad-\operatorname{Re}\left[\left(C_{L L R R}^{\omega}\right)_{m n p q}\left(C_{L L R R}^{\omega}\right)_{m n q p}^{*}\right]+\left|\left(C_{R R L L}^{\omega}\right)_{m n p q}\right|^{2} \\
& \quad+\left|\left(C_{R R L L}^{\omega}\right)_{m n q p}\right|^{2}-\operatorname{Re}\left[\left(C_{R R L L}^{\omega}\right)_{m n p q}\left(C_{R R L L}^{\omega}\right)_{m n q p}^{*}\right] \\
& \quad+\left|\left(C_{L R R L}^{\omega}\right)_{m n p q}\right|^{2}+\left|\left(C_{R L L R}^{\omega}\right)_{m n p q}\right|^{2}+\left|\left(C_{L R L R}^{\omega}\right)_{m n p q}\right|^{2} \\
& \left.\quad+\left|\left(C_{R L R L}^{\omega}\right)_{m n p q}\right|^{2}\right\},
\end{aligned}
$$

where $S=1$ (2) for $p=q \quad(p \neq q)$. The variable $\left(C_{R R R R}^{S_{1}}\right)_{m n p q}\left(\left(C_{R R R R}^{S_{2}}\right)_{m n p q}\right)$ corresponds to the contribution from $s_{1}^{+}\left(s_{2}^{+}\right)$in the first diagram (the second and the third diagrams) in Fig. 4: the structure of chiralities is $\ell_{m R} \rightarrow \bar{\ell}_{n R} \ell_{p R} \ell_{q R}$ because charged leptons that have Yukawa interactions with $s_{1}^{+}$and $s_{2}^{+}$are only right-handed ones. The contribution from $\eta^{+}$to $\ell_{m L} \rightarrow \bar{\ell}_{n L} \ell_{p L} \ell_{q L}$ via the second and the third diagrams in Fig. 4 is given by $\left(C_{L L L L}^{\eta}\right)_{m n p q}$. The other $\left(C^{\omega}\right)_{m n p q}$ 's arise due to the mixing between $s_{2}^{+}$and $\eta^{+}$in the second and the third diagrams in Fig. 4. See Appendix D for formulas of $(C)_{m n p q}$ 's. Current constraints on the branching ratios for LFV processes $\left(\ell \rightarrow \ell^{\prime} \gamma, h \rightarrow \ell \ell^{\prime}\right.$, and $\left.\ell_{m} \rightarrow \bar{\ell}_{n} \ell_{p} \ell_{q}\right)$ are summarized in Table II.

\section{DARK MATTER}

In our model, dark matter candidates are the lightest of fermions $\psi_{a}$ and a scalar $\eta^{0}$, which are neutral $Z_{2}$-odd particles. Notice that $\eta^{0}$ from a doublet field is a complex scalar with the lepton number $L=-1$. In other words, there is no mass splitting between $C P$-even and odd parts of $\eta^{0}$. According to Ref. [32], the scenario where the dark matter is such a complex scalar is stringently constrained from direct search experiments because it interacts with nuclei at tree level. Therefore, we consider the case where the dark matter is the lightest one of gauge singlet Majorana fermions $\psi_{a}$.

The dark matter candidate $\psi_{a}$ can be annihilated via treelevel diagrams shown in Fig. 5. The thermal averages

TABLE II. Current experimental constrains on branching ratios of LFV processes.

\begin{tabular}{lc}
\hline \hline Process & Upper limit \\
\hline$\mu \rightarrow e \gamma$ & $4.2 \times 10^{-13}[27]$ \\
$\tau \rightarrow e \gamma$ & $3.3 \times 10^{-8}[28]$ \\
$\tau \rightarrow \mu \gamma$ & $4.4 \times 10^{-8}[28]$ \\
\hline \hline Process & Upper limit \\
\hline$\mu \rightarrow \bar{e} e e$ & $1.0 \times 10^{-12}[29]$ \\
$\tau \rightarrow \bar{e} e e$ & $2.7 \times 10^{-8}[30]$ \\
$\tau \rightarrow \bar{\mu} e \mu$ & $2.7 \times 10^{-8}[30]$ \\
$\tau \rightarrow \bar{e} \mu \mu$ & $1.7 \times 10^{-8}[30]$ \\
$\tau \rightarrow \bar{e} e \mu$ & $1.8 \times 10^{-8}[30]$ \\
$\tau \rightarrow \bar{\mu} e e$ & $1.5 \times 10^{-8}[30]$ \\
$\tau \rightarrow \bar{\mu} \mu \mu$ & $2.1 \times 10^{-8}[30]$ \\
\hline \hline Process & Upper limit \\
\hline$h \rightarrow \mu e$ & $3.5 \times 10^{-4}[31]$ \\
$h \rightarrow \tau e$ & $6.1 \times 10^{-3}[22]$ \\
$h \rightarrow \mu \tau$ & $2.5 \times 10^{-3}[22]$ \\
\hline \hline
\end{tabular}




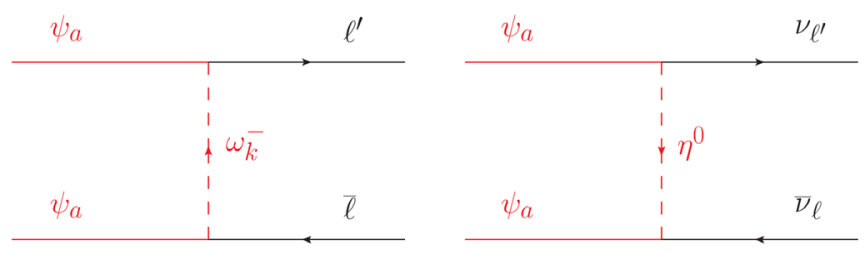

FIG. 5. Diagrams that give leading contributions to the DM relic abundance.

$\left\langle\sigma v_{\text {rel }}\right\rangle$, where $\sigma$ is annihilation cross section of $\psi_{a}$ and $v_{\text {rel }}$ denotes the relative velocity of the initial particles, is given by a sum of two processes as $\left\langle\sigma v_{\text {rel }}\right\rangle=\left\langle\sigma_{\ell} v_{\text {rel }}\right\rangle+\left\langle\sigma_{\nu} v_{\text {rel }}\right\rangle$. Thermal averages $\left\langle\sigma_{\ell} v_{\text {rel }}\right\rangle$ and $\left\langle\sigma_{\nu} v_{\text {rel }}\right\rangle$ correspond to the effects of left and right diagrams in Fig. 5, respectively. Formulas of $\left\langle\sigma_{\ell} v_{\text {rel }}\right\rangle$ and $\left\langle\sigma_{\nu} v_{\text {rel }}\right\rangle$ are shown in Appendix E. Notice that the $s$-wave annihilation is only involved in $\left\langle\sigma_{\ell} v_{\text {rel }}\right\rangle$ with a mixing $\chi$.

For the case where the elements of $Y_{\eta}$ and the mixing angle $\chi$ are negligible (we take such a benchmark scenario in the next section), the dominant contribution to $\left\langle\sigma v_{\text {rel }}\right\rangle$ comes from the mediation of $s_{2}^{+}\left(\simeq \omega_{2}^{+}\right)$in the left diagram in Fig. 5. Then, $\left\langle\sigma v_{\text {rel }}\right\rangle$ is approximately calculated as

$$
\left\langle\sigma v_{\mathrm{rel}}\right\rangle \simeq \frac{1}{8 \pi}\left(Y_{2}^{\dagger} Y_{2}\right)_{a a}^{2} \frac{M_{\psi_{a}}^{2}\left(M_{\psi_{a}}^{4}+m_{\omega_{2}}^{4}\right)}{\left(M_{\psi_{a}}^{2}+m_{\omega_{2}}^{2}\right)^{4}} \frac{1}{x}
$$

where $x=m_{\psi_{a}} / T$ at the temperature $T$. In Appendix E, $\left\langle\sigma v_{\text {rel }}\right\rangle$ for the more general case is presented. The relic abundance of $\psi_{a}$ with the $p$-wave annihilation is calculated as

$\Omega_{\psi_{a}} h^{2}=1.04 \times 10^{9} \times 2 \times x_{f}^{2} \frac{\sqrt{g_{*}}}{g_{* s}}\left\{\frac{\mathrm{GeV}^{-1}}{\left.m_{\mathrm{Pl}}\left\langle\sigma v_{\mathrm{rel}}\right\rangle\right|_{x=1}}\right\}$,

where $m_{\mathrm{Pl}}=1.2 \times 10^{19} \mathrm{GeV}$ stands for the Planck mass, and $g_{*}=106.75\left(g_{* S}=106.75\right)$ is the effective degree of freedom for energy (entropy) density in the era of the freeze out of the dark matter [33], and $x_{f}$ is defined by

$$
\begin{aligned}
x_{f}= & \ln \left[0.038 \times\left. 2\left(g_{\psi} / \sqrt{g_{*}}\right) m_{\mathrm{Pl}} M_{\psi_{a}}\left\langle\sigma v_{\text {rel }}\right\rangle\right|_{x=1}\right] \\
& -\frac{3}{2} \ln \left\{\ln \left[0.038 \times\left. 2\left(g_{\psi} / \sqrt{g_{*}}\right) m_{\mathrm{Pl}} M_{\psi_{a}}\left\langle\sigma v_{\text {rel }}\right\rangle\right|_{x=1}\right]\right\},
\end{aligned}
$$

where $g_{\psi}=2$ is the degree of freedom of $\psi_{a}$.

\section{BENCHMARK SCENARIOS AND NUMERICAL EVALUATION}

We here consider the possibility that $h \rightarrow \mu \tau$ is enhanced in comparison with LFV decays of charged leptons. First, we take the following benchmark scenario for $m_{1}<m_{3}$ (the normal ordering case of neutrino masses):

$$
\begin{aligned}
\sigma_{2} & =50 \mathrm{GeV}, \quad \sin 2 \theta \simeq-3.38 \times 10^{-2}, \quad \sin 2 \chi \simeq 4.99 \times 10^{-7}, \\
\lambda_{\phi 1} & =2.0, \quad \lambda_{\phi 2}=-2.0, \quad \lambda_{\phi \eta}=-2.0, \quad \lambda_{\phi \Phi}=\lambda_{\phi \Phi}^{\prime}=2.0, \\
M_{\psi_{1}} & =22.1 \mathrm{GeV}, \quad M_{\psi_{2}}=70 \mathrm{GeV}, \quad M_{\psi_{3}}=100 \mathrm{GeV}, \\
m_{\pi_{1}} & =520 \mathrm{GeV}, \quad m_{\pi_{2}}=510 \mathrm{GeV}, \\
m_{\omega_{1}}\left(m_{\eta^{+}}\right) & =1000 \mathrm{GeV}, \quad m_{\omega_{2}}\left(m_{s_{2}}\right)=550 \mathrm{GeV}, \quad m_{\eta^{0}}=1000 \mathrm{GeV}, \\
Y_{1} & =\left(\begin{array}{ccc}
10^{-4} & 10^{-4} & 0.10 \\
2.74 & 2.14 & 10^{-4} \\
3.50 & 3.47 & 10^{-4}
\end{array}\right), \\
Y_{2} & =\left(\begin{array}{ccc}
10^{-4} & 10^{-4} & 0.10 \\
-3.50 & -3.47 & 10^{-4} \\
2.26 & 3.50 & 10^{-4}
\end{array}\right), \quad Y_{\eta} \simeq\left(\begin{array}{ccc}
-1.17 \times 10^{-4} & -1.47 \times 10^{-5} & 4.17 \times 10^{-3} \\
1.90 \times 10^{-5} & -2.80 \times 10^{-5} & 1.83 \times 10^{-2} \\
1.25 \times 10^{-5} & 2.16 \times 10^{-5} & 2.16 \times 10^{-2}
\end{array}\right) .
\end{aligned}
$$

The small value of the mixing angle $\chi$ implies $\omega_{1}^{+} \simeq \eta^{+}$and $\omega_{2}^{+} \simeq s_{2}^{+}$. Since $\pi_{k}^{+}$and $\omega_{k}^{+}$have Yukawa interactions only with leptons, their masses are constrained by the slepton searches in the context of supersymmetric models at the LHC, which give about $500 \mathrm{GeV}$ as the lower bound [34].

The generated neutrino mass matrix results in the following values, which are consistent with the current constraint from neutrino oscillation experiments [26]:

$$
\begin{gathered}
\sin ^{2} \theta_{12}=0.307, \quad \sin ^{2} \theta_{13}=2.12 \times 10^{-2} \\
\sin ^{2} \theta_{23}=0.417, \\
\Delta m_{21}^{2}=7.53 \times 10^{-5} \mathrm{eV}^{2}, \\
\Delta m_{32}^{2}=2.51 \times 10^{-3} \mathrm{eV}^{2}, \\
m_{1}=0.048 \mathrm{eV}, \\
\delta=0,
\end{gathered}
$$


TABLE III. Numerical results for the LFV branching ratios in the benchmark scenario for the normal ordering case.

\begin{tabular}{lc}
\hline \hline Process & Numerical result \\
\hline$\mu \rightarrow e \gamma$ & $2.36 \times 10^{-15}$ \\
$\tau \rightarrow e \gamma$ & $8.26 \times 10^{-14}$ \\
$\tau \rightarrow \mu \gamma$ & $4.68 \times 10^{-10}$ \\
\hline \hline Process & Numerical result \\
\hline$\mu \rightarrow \bar{e} e e$ & $1.26 \times 10^{-18}$ \\
$\tau \rightarrow \bar{e} e e$ & $4.28 \times 10^{-18}$ \\
$\tau \rightarrow \bar{\mu} e \mu$ & $1.97 \times 10^{-11}$ \\
$\tau \rightarrow \bar{e} \mu \mu$ & $9.70 \times 10^{-12}$ \\
$\tau \rightarrow \bar{e} e \mu$ & $2.06 \times 10^{-10}$ \\
$\tau \rightarrow \bar{\mu} e e$ & $1.75 \times 10^{-12}$ \\
$\tau \rightarrow \bar{\mu} \mu \mu$ & $3.98 \times 10^{-11}$ \\
\hline \hline Process & \\
\hline$h \rightarrow \mu e$ & Numerical result \\
$h \rightarrow \tau e$ & $1.43 \times 10^{-16}$ \\
$h \rightarrow \mu \tau$ & $1.56 \times 10^{-15}$ \\
\hline \hline
\end{tabular}

where $\Delta m_{i j}^{2}=m_{i}^{2}-m_{j}^{2}$. The values of $m_{1}$ is also consistent with $\sum_{i} m_{i}<0.26 \mathrm{eV}$ that is given by cosmological observations [35], although $m_{1}$ is not constrained by the oscillation data.

In Table III, we show branching ratios for the LFV processes $\ell \rightarrow \ell^{\prime} \gamma, \ell_{m} \rightarrow \bar{\ell}_{n} \ell_{p} \ell_{q}$, and $h \rightarrow \ell \ell^{\prime}$ in our benchmark scenario given in Eq. (22). They satisfy the constraints from the current data in Table II. Since the elements of $Y_{\eta}$ are rather small as seen in Eq. (22), the contribution from $\eta^{+}$to $\ell \rightarrow \ell^{\prime} \gamma\left[A_{L}^{\omega}\right.$ in Eq. (16)] is negligible. Then, values of $\operatorname{BR}\left(\ell \rightarrow \ell^{\prime} \gamma\right)$ in our benchmark scenario are suppressed due to the cancellation of $A_{R}^{s 1}$ and $A_{R}^{\omega}$, which are contributions from $s_{1}^{+}$and $s_{2}^{+}$, respectively. This is an interesting utilization of scalar bosons $\left(s_{1}^{+}\right.$and $s_{2}^{+}$) that are originally introduced for generating neutrino masses. On the other hand, the contribution from $\eta^{+}$to $h \rightarrow \ell \ell^{\prime}\left[B_{L}^{\omega}\right.$ in Eq. (17)] is also negligible due to small values of components of $Y_{\eta}$. Even though contributions from $s_{1}^{+}$and $s_{2}^{+}$to $\ell \rightarrow \ell^{\prime} \gamma$ are destructive with each other, their contributions to $h \rightarrow \ell \ell^{\prime}\left[B_{R}^{s 1}\right.$ and $B_{R}^{\omega}$ in Eq. (17)] are not necessarily canceled with each other because of the sign flip by using coupling constants in the scalar sector, $\Lambda_{22}^{\pi}$ and $\Lambda_{22}^{\omega}$ in Appendix C. ${ }^{5}$ In our benchmark scenario, $\operatorname{BR}(h \rightarrow \mu \tau)$ is indeed much larger than $\operatorname{BR}(\tau \rightarrow \mu \gamma)$. This hierarchy is what expected in Ref. [23], and our calculation explicitly shows that the expectation is correct.

\footnotetext{
${ }^{5}$ Notice that other $\Lambda^{\pi}$ 's and $\Lambda^{\omega}$ 's do not contribute to the cancellation dominantly because $\theta$ and $\chi$ are small in the benchmark scenario.
}

In Fig. 6, we show plots of the branching ratio for $\tau \rightarrow \mu \gamma$ vs that for $h \rightarrow \mu \tau$. In the left one, we change only the values of $\left(Y_{1}\right)_{\mu 2}$ between -3.5 and 3.5. In the right one, we assume that the form of the matrices $Y_{1}$ and $Y_{2}$ is

$$
Y_{k}=\left(\begin{array}{ccc}
10^{-4} & 10^{-4} & 0.10 \\
\left(Y_{k}\right)_{\mu 1} & \left(Y_{k}\right)_{\mu 2} & 10^{-4} \\
\left(Y_{k}\right)_{\tau 1} & \left(Y_{k}\right)_{\tau 2} & 10^{-4}
\end{array}\right),
$$

where $k=1,2$, and then we vary eight unfixed parameters between -3.5 and 3.5. The orange points are predictions with same sign $\lambda$ 's, $\lambda_{\phi 1}=\lambda_{\phi 2}=\lambda_{\phi \eta}=\lambda_{\phi \Phi}=\lambda_{\phi \Phi}^{\prime}=2.0$. The blue points are ones with opposite sign $\lambda$ 's, $\lambda_{\phi 1}=$ $\lambda_{\phi \Phi}=\lambda_{\phi \Phi}^{\prime}=2.0$ and $\lambda_{\phi 2}=\lambda_{\phi \eta}=-2.0$, as in the benchmark scenario. In both of the plots, values of fixed parameters are taken to be the same with those of the benchmark scenario. Two branching ratios are equal on the solid line in the figures. The upper dashed line is the current upper limit for $\operatorname{BR}(\tau \rightarrow \mu \gamma), 4.4 \times 10^{-8}$, and the lower one is the expected upper limit, $1.0 \times 10^{-9}$, from the Belle-II experiment [36] with the integrated luminosity $50 \mathrm{ab}^{-1}$. In the case with same sign $\lambda$ 's, the correlation between branching ratios is almost linear, and $\operatorname{BR}(\tau \rightarrow \mu \gamma)$ is larger than $\operatorname{BR}(h \rightarrow \mu \tau)$ in most of the orange points. In the case with opposite sign $\lambda$ 's, on the other hand, $\mathrm{BR}(h \rightarrow \mu \tau)$ are significantly larger than $\operatorname{BR}(\tau \rightarrow \mu \gamma)$ in some of the blue points. This is just what we anticipated. The red point represents the result in the benchmark scenario.

In Fig. 7, we show the plot for $\mathrm{BR}(\tau \rightarrow \bar{\mu} \mu \mu)$ vs $\mathrm{BR}(h \rightarrow$ $\mu \tau)$ under the same assumptions as in the right one of Fig. 6. The upper dashed line is the current upper limit for $\operatorname{BR}(\tau \rightarrow \bar{\mu} \mu \mu), 2.1 \times 10^{-8}$, and the lower one is the expected upper limit, $3.3 \times 10^{-10}$, from the Belle-II experiment [36] with the integrated luminosity $50 \mathrm{ab}^{-1}$. We cannot find any correlation between the branching ratios, because these processes are given by different kinds of Feynman diagrams.

From Figs. 6 and 7, it is obvious that $\operatorname{BR}(\tau \rightarrow \mu \gamma)$ and $\operatorname{BR}(\tau \rightarrow \bar{\mu} \mu \mu)$ in our benchmark scenario is smaller than the each expected upper limits at the Belle II experiment, and these experiments cannot verify our scenario. However, the branching ratio of $h \rightarrow \mu \tau$ is about $10^{5}$-times larger than our prediction on $\operatorname{BR}(\tau \rightarrow \mu \gamma)$. The expected sensitivities of this process are $O\left(10^{-4}\right)$ at HL-LHC [37,38] and $O\left(10^{-5}\right)$ at ILC250 [39], and our prediction on $\operatorname{BR}(h \rightarrow$ $\mu \tau)$ is close to the sensitivity at ILC250. There is the possibility that we can test our scenario at ILC250 by detecting of $h \rightarrow \mu \tau$.

The dark matter in the benchmark scenario is the lightest $Z_{2}$-odd Majorana fermion $\psi_{1}$. The density of the thermal relic abundance $\Omega_{\psi_{1}} h^{2}$ can be evaluated with Eqs. (19)(21), which are valid for the case where $Y_{\eta}$ and $\chi$ are negligible. The Planck experiment shows that $\Omega_{\mathrm{DM}} h^{2}=$ $0.1200 \pm 0.0012$ [40]. In Fig. 8, we show $\Omega_{\psi_{1}} h^{2}$ as a 

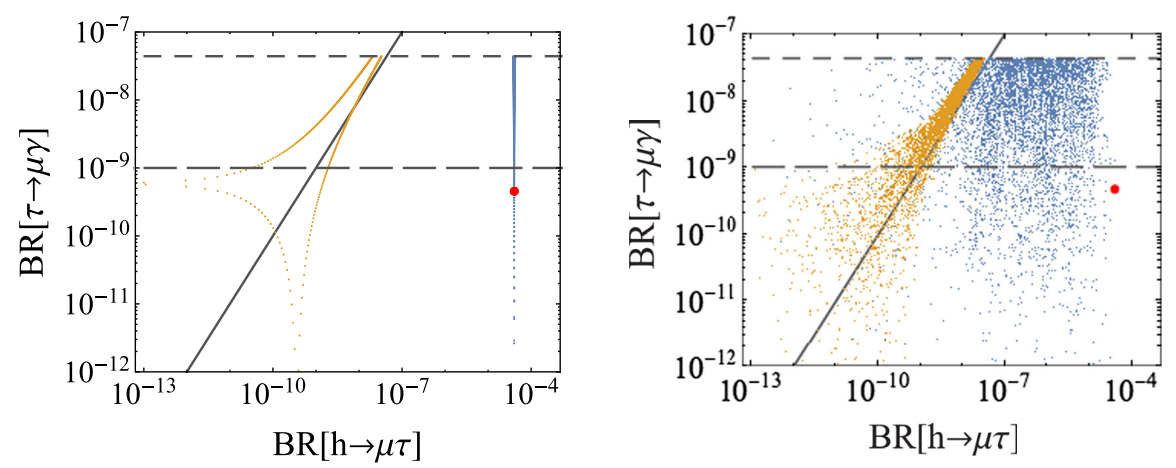

FIG. 6. Plots of the branching ratio for $\tau \rightarrow \mu \gamma$ vs that for $h \rightarrow \mu \tau$.

function of the dark matter mass $M_{\psi_{1}}$, where $Y_{2}$ and $m_{\omega}$ are fixed to the values of the benchmark scenario. The blue curve is the mass dependence in our model, and the horizontal line shows the observed value. It is clear that the appropriate value of $\Omega_{\psi_{1}} h^{2}$ is obtained for $M_{\psi_{1}}=$ $22.1 \mathrm{GeV}$ in the benchmark scenario.

There is no tree-level contribution to the dark matter scattering off nuclei, because $\psi_{a}$ are gauge singlet fermions. The scattering occurs at one-loop level via three penguin diagrams with $\omega_{1}, \omega_{2}$, and $\eta^{0}$ in the loop. In our benchmark scenario, the elements of the matrix $Y_{\eta}$ are typically smaller than those of $Y_{2}$, so that we consider only the contribution from the diagram with $\omega_{2}$ in the loop. In Ref. [41], the authors studied in detail the gauge singlet Majorana dark matter which is coupled to a dark scalar and charged leptons. They also considered the scenario where the dark matter has no interaction with electrons, which is similar to our benchmark scenario. They gave the constraint from the direct searches with the combined data from XENON1T [42], PandaX [43], and LUX [44]. From their results, we expect the dark matter in our benchmark scenario satisfies the constraint from the current direct detection experiments.

The Higgs boson can decay to a pair of dark matter particles in our benchmark scenario, and it is observed as

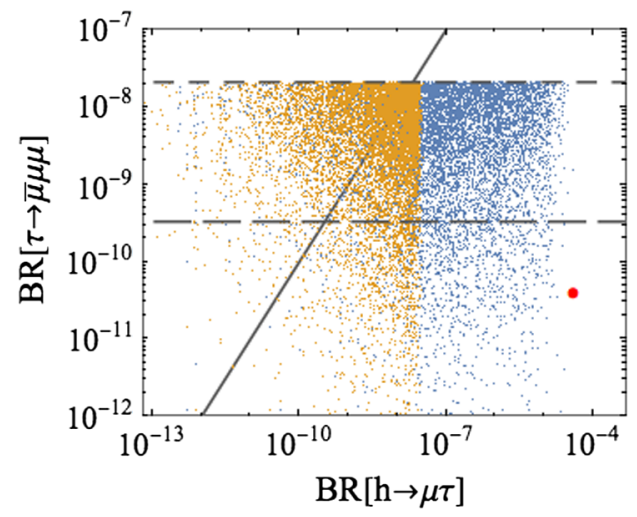

FIG. 7. The plot of the branching ratio for $\tau \rightarrow \bar{\mu} \mu \mu$ vs that for $h \rightarrow \mu \tau$. invisible decay of the Higgs boson at the collider experiments. The decay is induced via one-loop diagrams, and the branching ratio in our benchmark scenario is $\operatorname{BR}\left(h \rightarrow \psi_{1} \psi_{1}\right)=2.0 \times 10^{-3}$. The current upper limit for the branching ratio of $h \rightarrow$ inv is 0.24 [45]. Therefore, our benchmark scenario is consistent with this constraint. In our benchmark scenario, the $\mathrm{Z}$ boson can also decay to a pair of dark matter particles at one-loop level. The current data of the total invisible width of the $\mathrm{Z}$ boson from LEP is $499.0 \pm 1.5 \mathrm{MeV}$ [46], and the prediction in the $\mathrm{SM}$ of it is $501.44 \pm 0.04 \mathrm{MeV}$ [26]. These and the observed full decay width of the $\mathrm{Z}$ boson, $\Gamma=2.4952 \pm 0.0023 \mathrm{GeV}$ [46], lead the current upper limit for the branching ratio of $Z \rightarrow \psi_{1} \psi_{1}, \operatorname{BR}(Z \rightarrow$ $\left.\psi_{1} \psi_{1}\right)<2.0 \times 10^{-4}$. The prediction in our benchmark scenario is $\operatorname{BR}\left(Z \rightarrow \psi_{1} \psi_{1}\right)=1.2 \times 10^{-5}$, and it satisfies the current upper limit. The explicit formulas of $\Gamma(h \rightarrow$ $\left.\psi_{1} \psi_{1}\right)$ and $\Gamma\left(Z \rightarrow \psi_{1} \psi_{1}\right)$ in our benchmark scenario are shown in the Appendix F.

Next, we consider the benchmark scenario for the inverted ordering case $\left(m_{3}<m_{1}\right)$. The difference from

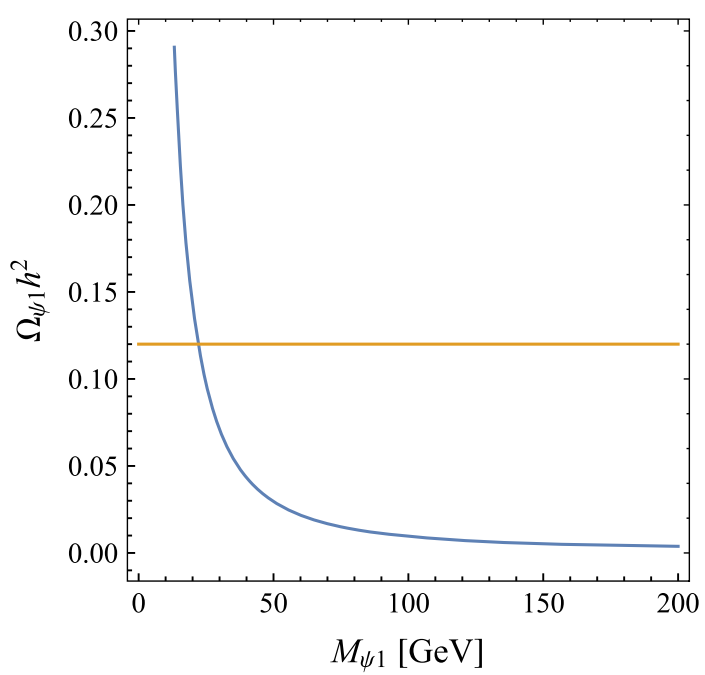

FIG. 8. Mass dependence of the DM relic abundance in the benchmark scenario for the normal ordering case of neutrino masses. 
the normal ordering case appears on $Y_{\eta}$, and we here take

$$
Y_{\eta} \simeq\left(\begin{array}{ccc}
-2.09 \times 10^{-4} & -2.54 \times 10^{-5} & 4.17 \times 10^{-3} \\
3.25 \times 10^{-5} & -4.76 \times 10^{-5} & 1.84 \times 10^{-2} \\
1.88 \times 10^{-5} & 4.05 \times 10^{-5} & 2.16 \times 10^{-2}
\end{array}\right)
$$

All the other parameters are taken to be the same with those in Eq. (22).

The neutrino mass matrix generated at two loop gives the following values, which are consistent with the current constraint from neutrino oscillation experiments [26],

$$
\begin{gathered}
\sin ^{2} \theta_{12}=0.307, \quad \sin ^{2} \theta_{13}=2.12 \times 10^{-2}, \\
\sin ^{2} \theta_{23}=0.421, \\
\Delta m_{21}^{2}=7.53 \times 10^{-5} \mathrm{eV}^{2}, \\
\Delta m_{32}^{2}=-2.56 \times 10^{-3} \mathrm{eV}^{2}, \\
m_{3}=0.07 \mathrm{eV}, \\
\delta=0 .
\end{gathered}
$$

The value of $m_{3}$ satisfies the condition from the Planck observation, $\sum_{i} m_{i}<0.26 \mathrm{eV}$ [35].

Branching ratios for the LFV processes in this scenario are listed in Table IV. All branching ratios are the same as

TABLE IV. Numerical results for the LFV branching ratios in the benchmark scenario for the inverted ordering case of neutrino masses.

\begin{tabular}{lc}
\hline \hline Process & Numerical result \\
\hline$\mu \rightarrow e \gamma$ & $2.36 \times 10^{-15}$ \\
$\tau \rightarrow e \gamma$ & $8.26 \times 10^{-14}$ \\
$\tau \rightarrow \mu \gamma$ & $4.68 \times 10^{-10}$ \\
\hline \hline Process & Numerical result \\
\hline$\mu \rightarrow \bar{e} e e$ & $1.26 \times 10^{-18}$ \\
$\tau \rightarrow \bar{e} e e$ & $4.28 \times 10^{-18}$ \\
$\tau \rightarrow \bar{\mu} e \mu$ & $1.97 \times 10^{-11}$ \\
$\tau \rightarrow \bar{e} \mu \mu$ & $9.70 \times 10^{-12}$ \\
$\tau \rightarrow \bar{e} e \mu$ & $2.06 \times 10^{-10}$ \\
$\tau \rightarrow \bar{\mu} e e$ & $1.75 \times 10^{-12}$ \\
$\tau \rightarrow \bar{\mu} \mu \mu$ & $3.98 \times 10^{-11}$ \\
\hline \hline Process & \\
\hline$h \rightarrow \mu e$ & Numerical result \\
$h \rightarrow \tau e$ & $1.43 \times 10^{-16}$ \\
$h \rightarrow \mu \tau$ & $1.56 \times 10^{-15}$ \\
\hline \hline
\end{tabular}

those in the scenario in Eq. (22), because the elements of $Y_{\eta}$ are typically smaller than those of $Y_{1}$ and $Y_{2}$ in the both scenarios. $\operatorname{BR}(h \rightarrow \mu \tau)$ is about $10^{5}$ times larger than our prediction on $\operatorname{BR}(\tau \rightarrow \mu \gamma)$.

The dark matter in this scenario is again the lightest $Z_{2}$-odd Majorana fermion $\psi_{1}$. The density of the thermal relic abundance depends on only $\left(Y_{2}^{\dagger} Y_{2}\right)_{11}, M_{\psi_{1}}$ and $m_{\omega_{2}}$ in the case, where $Y_{\eta}$ and $\chi$ are negligibly small. Values of these parameters are the same with those of Eq. (22). Therefore, $M_{\psi_{1}}=22.1 \mathrm{GeV}$ can still explain the observed relic density $\Omega_{\mathrm{DM}} h^{2}=0.1200 \pm 0.0012$ [40], just like in the benchmark scenario for the case of $m_{1}<m_{3}$. The constraints from the direct detection experiments and the invisible decay of the Higgs boson and the $\mathrm{Z}$ boson are also the same with those in the previous scenario.

\section{CONCLUSIONS}

We have proposed a new mechanism to explain neutrino masses with lepton number conservation, in which the Dirac neutrino masses are generated at the two-loop level involving a dark matter candidate. In this model branching ratios of lepton flavor violating decays of the Higgs boson can be much larger than those of lepton flavor violating decays of charged leptons. We have found the benchmark scenarios for normal ordered masses of neutrinos and inverted ones, where the neutrino mass matrix, the relic density of dark matter and the branching ratios for LFV processes can satisfy the constraints from current experimental data. We have showed that $\operatorname{BR}(h \rightarrow \mu \tau)$ is about $10^{5}$ lager than $\operatorname{BR}(\tau \rightarrow \mu \gamma)$ in our benchmark scenarios. If the lepton flavor violating decays of the Higgs boson are observed at the future collider experiments without detecting lepton flavor violating decays of charged leptons, most of the previously proposed models are excluded, while our model can still survive.

In this paper, we did not discuss collider signature of new scalars and fermions. Collider phenomenology for $Z_{2}$-even/odd charged singlet scalars in different models can be found in the literature $[47,48] /[48-50]$, while that for $\Phi$ $(Y=3 / 2)$ has been discussed in Refs. [51,52] in the different context. We will discuss these issues elsewhere in the future [53].

\section{ACKNOWLEDGMENTS}

The work of K.E. was supported in part by the Sasakawa Scientific Research Grant from The Japan Science Society. The work of S. K. was supported in part by Grant-in-Aid for Scientific Research on Innovative Areas, the Ministry of Education, Culture, Sports, Science and Technology (MEXT), No. 16H06492, No. 18H04587, and Grant H2020-MSCA-RISE-2014 No. 645722 (Non Minimal Higgs). The work of K. S. was supported in part by Japan Society for the Promotion of Science (JSPS) KAKENHI Grant No. 18J12866 
(JSPS Research Fellow). The work of H. S. was supported in part by MEXT KAKENHI Grant No. 18H05543 (Innovative Areas) and JSPS KAKENHI Grant No. 18K03625 [Scientific Research (C)].

\section{APPENDIX A: THE LOOP FUNCTION IN THE NEUTRINO MASS MATRIX}

The neutrino mass matrix formula given by Eq. (13) in Sec. III contains the loop function $I_{\ell^{\prime} a k}(k=1,2, a=1,2$, 3 and $\left.\ell^{\prime}=e, \mu, \tau\right)$. We here show the explicit formula for $I_{\ell^{\prime} a k}$;

$$
\begin{aligned}
I_{\ell^{\prime} a k}= & \frac{1}{\left(16 \pi^{2}\right)^{2}} \frac{1}{\left(m_{\pi_{2}}^{2}-m_{\pi_{1}}^{2}\right)\left(M_{\psi_{a}}^{2}-m_{\eta^{0}}^{2}\right)} \\
& \times \int_{0}^{1} \mathrm{~d} z z\left\{\frac{1}{m_{\pi_{1}}^{2}-m_{\ell^{\prime}}^{2}}\left(f_{a k}\left(m_{\pi_{1}}^{2}\right)-f_{a k}\left(m_{\ell^{\prime}}^{2}\right)\right)\right. \\
& \left.-\frac{1}{m_{\pi_{2}}^{2}-m_{\ell^{\prime}}^{2}}\left(f_{a k}\left(m_{\pi_{2}}^{2}\right)-f_{a k}\left(m_{\ell^{\prime}}^{2}\right)\right)\right\}
\end{aligned}
$$

where the function $f_{a k}$ is defined as follows

$$
f_{a k}\left(m^{2}\right)=m^{4}\left\{\operatorname{Li}_{2}\left(z_{k a}^{\psi}\left(m^{2}\right)\right)-\operatorname{Li}_{2}\left(z_{k}^{\eta}\left(m^{2}\right)\right)\right\},
$$

with

$$
\begin{gathered}
z_{a k}^{\psi}\left(m^{2}\right)=1-\frac{1}{z(1-z) m^{2}}\left\{M_{\psi_{a}}^{2}+z\left(m_{\omega_{k}}^{2}-M_{\psi_{a}}^{2}\right)\right\}, \\
z_{k}^{\eta}\left(m^{2}\right)=1-\frac{1}{z(1-z) m^{2}}\left\{m_{\eta^{0}}^{2}+z\left(m_{\omega_{k}}^{2}-m_{\eta^{0}}^{2}\right)\right\}, \\
\operatorname{Li}_{2}(x)=\int_{0}^{x} \mathrm{~d} t \frac{1}{-t} \ln (1-t) .
\end{gathered}
$$

\section{APPENDIX B: SOME FORMULAS FOR $\ell \rightarrow \ell^{\prime} \gamma$}

In Sec. IV, blanching ratios for $\ell \rightarrow \ell^{\prime} \gamma$ are given by Eq. (16), which depend on $A_{R}^{s_{1}}, A_{R}^{\omega}$ and $A_{L}^{\omega}$. We here present their explicit formulas. They are given by

$$
\begin{gathered}
A_{R}^{s_{1}}=\sum_{k} \frac{1}{12} \frac{m_{\ell}^{2}}{m_{\pi_{k}}^{2}}\left(Y_{1} Y_{1}^{\dagger}\right)_{\ell \ell^{\prime}}\left(U_{\theta}\right)_{k 2}^{2}, \\
A_{R}^{\omega}=\sum_{a, k} \frac{1}{2} \frac{m_{\ell}^{2}}{m_{\omega_{k}}^{2}}\left[\left(Y_{2}\right)_{\ell^{\prime} a}^{*}\left(Y_{2}\right)_{\ell a}\left(U_{\chi}\right)_{k 2}^{2} F_{2}\left(\frac{M_{\psi_{a}}^{2}}{m_{\omega_{k}}^{2}}\right)\right. \\
\left.-\frac{M_{\psi_{a}}}{m_{\ell}}\left(Y_{2}\right)_{\ell^{\prime} a}^{*}\left(Y_{\eta}\right)_{\ell a}^{*}\left(U_{\chi}\right)_{k 1}\left(U_{\chi}\right)_{k 2} G\left(\frac{M_{\psi_{a}}^{2}}{m_{\omega_{k}}^{2}}\right)\right],
\end{gathered}
$$

$$
\begin{aligned}
A_{L}^{\omega}= & \sum_{a, k} \frac{1}{2} \frac{m_{\ell}^{2}}{m_{\omega_{k}}^{2}}\left[\left(Y_{\eta}\right)_{\ell^{\prime} a}\left(Y_{\eta}\right)_{\ell a}^{*}\left(\chi_{k}^{\prime}\right)^{2} F_{2}\left(\frac{M_{\psi_{a}}^{2}}{m_{\omega_{k}}^{2}}\right)\right. \\
& \left.-\frac{M_{\psi_{a}}}{m_{\ell}}\left(Y_{2}\right)_{\ell a}\left(Y_{\eta}\right)_{\ell^{\prime} a}\left(U_{\chi}\right)_{k 1}\left(U_{\chi}\right)_{k 2} G\left(\frac{M_{\psi_{a}}^{2}}{m_{\omega_{k}}^{2}}\right)\right],
\end{aligned}
$$

where $F_{2}(x)$ and $G(x)$ are defined as

$$
\begin{gathered}
F_{2}(x)=\frac{1}{6(1-x)^{4}}\left(1-6 x+3 x^{2}+2 x^{3}-6 x^{2} \ln x\right), \\
G(x)=\frac{1}{(1-x)^{3}}\left(1-x^{2}+2 x \ln x\right) .
\end{gathered}
$$

Terms that proportional to $M_{\psi_{a}} / m_{\ell}$ in formulas of $A_{R}^{\omega}$ and $A_{L}^{\omega}$ appear due to the mixing between $s_{2}^{+}$and $\eta^{+}$.

\section{APPENDIX C: SOME FORMULAS FOR $\boldsymbol{h} \rightarrow \boldsymbol{\ell} \boldsymbol{\ell}^{\prime}$}

In Sec. IV, blanching ratios for $h \rightarrow \ell \ell^{\prime}$ are given by Eq. (17), which depend on $B_{R}^{s_{1}}, B_{R}^{\omega}$, and $B_{L}^{\omega}$. We here give their explicit formulas. They are defined as

$$
\begin{aligned}
B_{R}^{S_{1}}= & m_{\ell}\left(Y_{1} Y_{1}^{\dagger}\right)_{\ell \ell^{\prime}} \sum_{k, k^{\prime}} \Lambda_{k k^{\prime}}^{\pi}\left(U_{\theta}\right)_{k 2}\left(U_{\theta}\right)_{k^{\prime} 2} \\
& \times \int_{0}^{1} \mathrm{~d} x \mathrm{~d} y \mathrm{~d} z \frac{z}{y m_{\pi_{k}}^{2}+z m_{\pi_{k^{\prime}}}^{2}-y z m_{h}^{2}},
\end{aligned}
$$

$$
\begin{aligned}
B_{R}^{\omega}= & \sum_{a, k, k^{\prime}} m_{\ell}\left(Y_{2}\right)_{\ell a}\left(Y_{2}\right)_{\ell^{\prime} a}^{*} \Lambda_{k k^{\prime}}^{\omega}\left(U_{\chi}\right)_{k 2}\left(U_{\chi}\right)_{k^{\prime} 2} \\
& \times \int_{0}^{1} \mathrm{~d} x \mathrm{~d} y \mathrm{~d} z \frac{z}{x M_{\psi_{a}}^{2}+y m_{\omega_{k}}^{2}+z m_{\omega_{k^{\prime}}}^{2}-y z m_{h}^{2}} \\
& +\sum_{a, k, k^{\prime}} M_{\psi_{a}}\left(Y_{\eta}\right)_{\ell a}^{*}\left(Y_{2}\right)_{\ell^{\prime} a}^{*} \Lambda_{k k^{\prime}}^{\omega}\left(U_{\chi}\right)_{k 1}\left(U_{\chi}\right)_{k^{\prime} 2} \\
& \times \int_{0}^{1} \mathrm{~d} x \mathrm{~d} y \mathrm{~d} z \frac{1}{x M_{\psi_{a}}^{2}+y m_{\omega_{k}}^{2}+z m_{\omega_{k^{\prime}}}^{2}-y z m_{h}^{2}}, \\
B_{L}^{\omega}= & \sum_{a, k, k^{\prime}} m_{\ell}\left(Y_{\eta}\right)_{\ell a}^{*}\left(Y_{\eta}\right)_{\ell^{\prime} a} \Lambda_{k k^{\prime}}^{\omega}\left(U_{\chi}\right)_{k 1}\left(U_{\chi}\right)_{k^{\prime} 1} \\
& \times \int_{0}^{1} \mathrm{~d} x \mathrm{~d} y \mathrm{~d} z \frac{z}{x M_{\psi_{a}}^{2}+y m_{\omega_{k}}^{2}+z m_{\omega_{k^{\prime}}}^{2}-y z m_{h}^{2}} \\
& +\sum_{a, k, k^{\prime}} M_{\psi_{a}}\left(Y_{2}\right)_{\ell a}\left(Y_{\eta}\right)_{\ell^{\prime} a} \Lambda_{k k^{\prime}}^{\omega}\left(U_{\chi}\right)_{k 2}\left(U_{\chi}\right)_{k^{\prime} 1} \\
& \times \int_{0}^{1} \mathrm{~d} x \mathrm{~d} y \mathrm{~d} z \frac{1}{x M_{\psi_{a}}^{2}+y m_{\omega_{k}}^{2}+z m_{\omega_{k^{\prime}}}^{2}-y z m_{h}^{2}} .
\end{aligned}
$$

Coefficients $\Lambda_{k k^{\prime}}^{\pi}$ and $\Lambda_{k k^{\prime}}^{\omega}$ are defined in order to satisfy

$$
\mathcal{L}=\sum_{k, k^{\prime}}\left(\Lambda_{k k^{\prime}}^{\pi} \pi_{k}^{+} \pi_{k^{\prime}}^{-}+\Lambda_{k k^{\prime}}^{\omega} \omega_{k}^{+} \omega_{k^{\prime}}^{-}\right) h,
$$


and given by

$\Lambda_{11}^{\pi}=-\frac{\sigma_{1}}{\sqrt{2}} \sin 2 \theta-\left(\lambda_{\phi \Phi}+\lambda_{\phi \Phi}^{\prime}\right) v \cos ^{2} \theta-\lambda_{\phi 1} v \sin ^{2} \theta$,

$\Lambda_{12}^{\pi}=\Lambda_{21}^{\pi}=-\frac{\sigma_{1}}{\sqrt{2}} \cos 2 \theta+\frac{1}{2}\left(\lambda_{\phi \Phi}+\lambda_{\phi \Phi}^{\prime}\right) v \sin 2 \theta$

$$
-\frac{1}{2} \lambda_{\phi 1} v \sin 2 \theta \text {, }
$$

$\Lambda_{22}^{\pi}=\frac{\sigma_{1}}{\sqrt{2}} \sin 2 \theta-\left(\lambda_{\phi \Phi}+\lambda_{\phi \Phi}^{\prime}\right) v \sin ^{2} \theta-\lambda_{\phi 1} v \cos ^{2} \theta$

$$
\Lambda_{11}^{\omega}=\frac{\sigma_{3}}{\sqrt{2}} \sin 2 \chi-\lambda_{\phi \eta} v \cos ^{2} \chi-\lambda_{\phi 2} v \sin ^{2} \chi
$$

$$
\Lambda_{12}^{\omega}=\Lambda_{21}^{\omega}=\frac{\sigma_{3}}{\sqrt{2}} \cos 2 \chi+\frac{1}{2} \lambda_{\phi \eta} v \sin 2 \chi-\frac{1}{2} \lambda_{\phi 2} v \sin 2 \chi,
$$

$$
\Lambda_{22}^{\omega}=-\frac{\sigma_{3}}{\sqrt{2}} \sin 2 \chi-\lambda_{\phi \eta} v \sin ^{2} \chi-\lambda_{\phi 2} v \cos ^{2} \chi
$$

\section{APPENDIX D: SOME FORMULAS FOR $\ell_{m} \rightarrow \bar{\ell}_{n} \ell_{p} \ell_{q}$}

In Sec. IV, blanching ratios for $\ell_{m} \rightarrow \bar{\ell}_{n} \ell_{p} \ell_{q}$ are given by Eq. (18), which depend on $\left(C_{R R R R}^{s_{1}}\right)_{m n p q},\left(C_{R R R R}^{s_{2}}\right)_{m n p q}$, $\left(C^{\eta}\right)_{m n p q}$, and $\left(C^{\omega}\right)_{m n p q}^{\prime} \mathrm{s}$. We here give their explicit formulas. They are given by

$$
\begin{aligned}
& \left(C_{R R R R}^{S_{1}}\right)_{m n p q}=-\frac{1}{2}\left[\left(Y_{1} Y_{1}^{\dagger}\right)_{m p}\left(Y_{1} Y_{1}^{\dagger}\right)_{n q}+(p \leftrightarrow q)\right] \sum_{k, k^{\prime}}\left(U_{\theta}\right)_{k 2}^{2}\left(U_{\theta}\right)_{k^{\prime} 2}^{2} \int \frac{1}{\Delta} \\
& \left(C_{R R R R}^{s_{2}}\right)_{m n p q}=-\sum_{a, b, k, k^{\prime}} \frac{1}{2}\left(\left(Y_{2}\right)_{m a}\left(Y_{2}\right)_{n b}\left(Y_{2}\right)_{p a}^{*}\left(Y_{2}\right)_{q b}^{*}+(p \leftrightarrow q)\right)\left(U_{\chi}\right)_{k 2}^{2}\left(U_{\chi}\right)_{k^{\prime} 2}^{2} \int \frac{1}{\Sigma} \\
& -\sum_{a, b, k, k^{\prime}} M_{\psi_{a}} M_{\psi_{b}}\left(Y_{2}\right)_{m a}\left(Y_{2}\right)_{n a}\left(Y_{2}\right)_{p b}^{*}\left(Y_{2}\right)_{q b}^{*}\left(U_{\chi}\right)_{k 2}^{2}\left(U_{\chi}\right)_{k^{\prime} 2}^{2} \int \frac{1}{\Sigma^{2}} \\
& \left(C_{L L L L}^{\eta}\right)_{m n p q}=-\sum_{a, b, k, k^{\prime}} \frac{1}{2}\left(\left(Y_{\eta}\right)_{m a}^{*}\left(Y_{\eta}\right)_{n b}^{*}\left(Y_{\eta}\right)_{p a}\left(Y_{\eta}\right)_{q b}+(p \leftrightarrow q)\right)\left(U_{\chi}\right)_{k 1}^{2}\left(U_{\chi}\right)_{k^{\prime} 1}^{2} \int \frac{1}{\Sigma} \\
& -\sum_{a, b, k, k^{\prime}} M_{\psi_{a}} M_{\psi_{b}}\left(Y_{\eta}\right)_{m a}^{*}\left(Y_{\eta}\right)_{n a}^{*}\left(Y_{\eta}\right)_{p b}\left(Y_{\eta}\right)_{q b}\left(U_{\chi}\right)_{k 1}^{2}\left(U_{\chi}\right)_{k^{\prime} 1}^{2} \int \frac{1}{\Sigma^{2}} \\
& \left(C_{L L R R}^{\omega}\right)_{m n p q}=\sum_{a, b, k, k^{\prime}} M_{\psi_{a}} M_{\psi_{b}}\left(Y_{\eta}\right)_{m a}^{*}\left(Y_{\eta}\right)_{n b}^{*}\left(Y_{2}\right)_{p a}^{*}\left(Y_{2}\right)_{q b}^{*}\left(U_{\chi}\right)_{k 1}\left(U_{\chi}\right)_{k^{\prime} 1}\left(U_{\chi}\right)_{k 2}\left(U_{\chi}\right)_{k^{\prime} 2} \int \frac{1}{\Sigma^{2}}, \\
& \left(C_{R R L L}^{\omega}\right)_{m n p q}=\sum_{a, b, k, k^{\prime}} M_{\psi_{a}} M_{\psi_{b}}\left(Y_{2}\right)_{m a}\left(Y_{2}\right)_{n b}\left(Y_{\eta}\right)_{p a}\left(Y_{\eta}\right)_{q b}\left(U_{\chi}\right)_{k 2}\left(U_{\chi}\right)_{k^{\prime} 2}\left(U_{\chi}\right)_{k 1}\left(U_{\chi}\right)_{k^{\prime} 1} \int \frac{1}{\Sigma^{2}}, \\
& \left(C_{R L L R}^{\omega}\right)_{m n p q}=\sum_{a, b, k, k^{\prime}}\left(Y_{2}\right)_{m a}\left(Y_{\eta}\right)_{n b}^{*}\left(U_{\chi}\right)_{k 2}\left(U_{\chi}\right)_{k^{\prime} 1} \\
& \times\left(M_{\psi_{a}} M_{\psi_{b}}\left(Y_{\eta}\right)_{p a}\left(Y_{2}\right)_{q b}^{*}\left(U_{\chi}\right)_{k^{\prime} 1}\left(U_{\chi}\right)_{k 2} \int \frac{1}{\Sigma^{2}}+\left(Y_{\eta}\right)_{p b}\left(Y_{2}\right)_{q a}^{*}\left(U_{\chi}\right)_{k 1}\left(U_{\chi}\right)_{k^{\prime} 2} \int \frac{1}{\Sigma}\right) \\
& -\sum_{a, b, k, k^{\prime}}\left(Y_{2}\right)_{m a}\left(Y_{\eta}\right)_{n a}^{*}\left(U_{\chi}\right)_{k 2}\left(U_{\chi}\right)_{k^{\prime} 1}\left(Y_{\eta}\right)_{p b}\left(Y_{2}\right)_{q b}^{*}\left(\left(U_{\chi}\right)_{k 1}\left(U_{\chi}\right)_{k^{\prime} 2}-\left(U_{\chi}\right)_{k^{\prime} 1}\left(U_{\chi}\right)_{k 2}\right) \int \frac{1}{\Sigma}, \\
& \left(C_{L R R L}^{\omega}\right)_{m n p q}=\sum_{a, b, k, k^{\prime}}\left(Y_{\eta}\right)_{m a}^{*}\left(Y_{2}\right)_{n b}\left(U_{\chi}\right)_{k 1}\left(U_{\chi}\right)_{k^{\prime} 2} \\
& \times\left(M_{\psi_{a}} M_{\psi_{b}}\left(Y_{2}\right)_{p a}^{*}\left(Y_{\eta}\right)_{q b}\left(U_{\chi}\right)_{k 1}\left(U_{\chi}\right)_{k^{\prime} 2} \int \frac{1}{\Sigma^{2}}+\left(Y_{2}\right)_{p b}^{*}\left(Y_{\eta}\right)_{q a}\left(U_{\chi}\right)_{k 2}\left(U_{\chi}\right)_{k^{\prime} 1} \int \frac{1}{\Sigma}\right) \\
& -\sum_{a, b, k, k^{\prime}}\left(Y_{\eta}\right)_{m a}^{*}\left(Y_{2}\right)_{n a}\left(U_{\chi}\right)_{k 1}\left(U_{\chi}\right)_{k^{\prime} 2}\left(Y_{2}\right)_{p b}^{*}\left(Y_{\eta}\right)_{q b}\left(\left(U_{\chi}\right)_{k 2}\left(U_{\chi}\right)_{k^{\prime} 1}-\left(U_{\chi}\right)_{k 1}\left(U_{\chi}\right)_{k^{\prime} 2}\right) \int \frac{1}{\Sigma} \text {, }
\end{aligned}
$$


where $\Delta$ and $\Sigma$ are defined as

$$
\begin{gathered}
\Delta=x m_{\pi_{k}}^{2}+y m_{\pi_{k^{\prime}}}^{2} \\
\Sigma=x m_{\omega_{k}}^{2}+y m_{\omega_{k^{\prime}}}^{2}+z M_{\psi_{a}}^{2}+\omega M_{\psi_{b}}^{2},
\end{gathered}
$$

and the symbol $\int$ denotes the integration with respect to $x$, $y, z$, and $\omega$ as follows;

$$
\int=\int_{0}^{1} \mathrm{~d} x \mathrm{~d} y \mathrm{~d} z \mathrm{~d} \omega .
$$

By exchanging $p$ and $q$ for $\left(C_{R L L R}^{\omega}\right)_{m n p q}$ and $\left(C_{L R R L}^{\omega}\right)_{m n p q}$, we obtain

$$
\begin{aligned}
& \left(C_{R L R L}^{\omega}\right)_{m n p q}=-\left(C_{R L L R}^{\omega}\right)_{m n q p}, \\
& \left(C_{L R L R}^{\omega}\right)_{m n p q}=-\left(C_{L R R L}^{\omega}\right)_{m n q p} .
\end{aligned}
$$

\section{APPENDIX E: ANNIHILATION OF DARK MATTER $\psi_{a}$}

In Sec. V, we have shown only the approximate formula for the thermal averaged cross section for annihilation of the dark matter $\psi_{a},\left\langle\sigma v_{\text {rel }}\right\rangle$. In this Appendix, we show the complete formula at tree level. First, the contribution from annihilation to a pair of charged leptons, $\left\langle\sigma_{\ell} v_{\text {rel }}\right\rangle$, which is shown by the left of Fig. 5, is given by

$$
\begin{aligned}
\left\langle\sigma_{\ell} v_{\text {rel }}\right\rangle= & \sum_{k, k^{\prime}} \frac{1}{8 \pi}\left(\left(Y_{2}^{\dagger} Y_{2}\right)_{a a}^{2}\left(U_{\chi}\right)_{k 2}^{2}\left(U_{\chi}\right)_{k^{\prime} 2}^{2}+\left(Y_{\eta}^{\dagger} Y_{\eta}\right)_{a a}^{2}\left(U_{\chi}\right)_{k 1}^{2}\left(U_{\chi}\right)_{k^{\prime} 1}^{2}\right) \\
& \times \frac{M_{\psi_{a}}^{2}\left(M_{\psi_{a}}^{4}+m_{\omega_{k}}^{2} m_{\omega_{k^{\prime}}}^{2}\right)}{\left(M_{\psi_{a}}^{2}+m_{\omega_{k}}^{2}\right)^{2}\left(M_{\psi_{a}}^{2}+m_{\omega_{k^{\prime}}}^{2}\right)^{2}} \frac{1}{x} \\
& +\sum_{k, k^{\prime}} \frac{1}{16 \pi}\left(Y_{2}^{\dagger} Y_{2}\right)_{a a}\left(Y_{\eta}^{\dagger} Y_{\eta}\right)_{a a}\left(U_{\chi}\right)_{k 2}^{2}\left(U_{\chi}\right)_{k^{\prime} 2}^{2}\left(U_{\chi}\right)_{k 1}^{2}\left(U_{\chi}\right)_{k^{\prime} 1}^{2} \\
& \times\left[\frac{2 M_{\psi_{a}}^{2}}{\left(M_{\psi_{a}}^{2}+m_{\omega_{k}}^{2}\right)\left(M_{\psi_{a}}^{2}+m_{\omega_{k^{\prime}}}^{2}\right)}+\frac{M_{\psi_{a}}^{2}}{\left(M_{\psi_{a}}^{2}+m_{\omega_{k}}^{2}\right)^{3}\left(M_{\psi_{a}}^{2}+m_{\omega_{k^{\prime}}}^{2}\right)^{3}}\right. \\
& \times\left\{5 M_{\psi_{a}}^{8}+12 M_{\psi_{a}}^{6}\left(m_{\omega_{k}}^{2}+m_{\omega_{k^{\prime}}}^{2}\right)+3 M_{\psi_{a}}^{4}\left(m_{\omega_{k}}^{4}+8 m_{\omega_{k}}^{2} m_{\omega_{k^{\prime}}}^{2}+m_{\omega_{k^{\prime}}}^{4}\right)\right. \\
& \left.\left.+4 M_{\psi_{a}}^{2} m_{\omega_{k}}^{2} m_{\omega_{k^{\prime}}}^{2}\left(m_{\omega_{k}}^{2}+m_{\omega_{k^{\prime}}}^{2}\right)-3 m_{\omega_{k}}^{4} m_{\omega_{k^{\prime}}}^{4}\right\} \frac{1}{x}\right] .
\end{aligned}
$$

Second, the contribution from annihilation to a pair of neutrinos, $\left\langle\sigma_{\nu} v_{\text {rel }}\right\rangle$, which is represented by the right of Fig. 5, is given by

$$
\left\langle\sigma_{\nu} v_{\mathrm{rel}}\right\rangle=\frac{\left(Y_{\eta}^{\dagger} Y_{\eta}\right)_{a a}^{2}}{8 \pi} \frac{M_{\psi_{a}}^{2}\left(M_{\psi_{a}}^{4}+m_{\eta}^{4}\right)}{\left(M_{\psi_{a}}^{2}+m_{\eta}^{2}\right)^{4}} \frac{1}{x} .
$$

The complete formula for $\left\langle\sigma v_{\text {rel }}\right\rangle$ is given at tree level by the sum of Eq. (E1) and (E2).

\section{APPENDIX F: DECAY RATES OF THE INVISIBLE DECAY OF THE HIGGS BOSON AND THE $Z$ BOSON}

In the benchmark scenarios in Sec. VI, the Higgs boson and the $\mathrm{Z}$ boson can decay to a pair of dark matters. In this Appendix, we show the formulas of $\Gamma\left(h \rightarrow \psi_{1} \psi_{1}\right)$ and $\Gamma\left(Z \rightarrow \psi_{1} \psi_{1}\right)$. We assume the elements of $Y_{\eta}$ and the mixing angle $\chi$ are negligibly small as the benchmark scenarios, and the only leading terms of the each decay rates are shown. The decay rate of the Higgs boson to a pair of dark matters is given by
$\Gamma\left(h \rightarrow \psi_{1} \psi_{1}\right)=\frac{m_{h}}{4 \pi}\left(\frac{1}{16 \pi^{2}}\right)^{2}\left(1-4 \frac{M_{\psi_{1}}^{2}}{m_{h}^{2}}\right)^{\frac{3}{2}}\left(\lambda_{\phi 2} v\right)^{2}\left|D_{h}\right|^{2}$,

where

$$
\begin{aligned}
D_{h}= & \sum_{\ell=e, \mu, \tau}\left|\left(Y_{2}\right)_{\ell 1}\right|^{2} \int_{0}^{1} \mathrm{~d} x \mathrm{~d} y \mathrm{~d} z \\
& \times \frac{z M_{\psi_{1}}}{y m_{\ell}^{2}+(1-y) m_{\omega_{2}}^{2}-y(1-y) M_{\psi_{1}}^{2}-x z m_{h}^{2}} .
\end{aligned}
$$

The decay rate of the $\mathrm{Z}$ boson to a pair of dark matters is given by

$\Gamma\left(Z \rightarrow \psi_{1} \psi_{1}\right)=\frac{m_{Z} \alpha \tan ^{2} \theta_{\mathrm{w}}}{6}\left(\frac{1}{16 \pi^{2}}\right)^{2}\left(1-4 \frac{M_{\psi_{1}}^{2}}{m_{Z}^{2}}\right)^{\frac{3}{2}}\left|D_{Z}\right|^{2}$,

where $m_{Z}$ is the mass of the $Z$ boson, $\theta_{\mathrm{w}}$ is the Weinberg angle and $D_{Z}$ is given by 


$$
\begin{aligned}
D_{Z}= & \sum_{\ell=e, \mu, \tau}\left|\left(Y_{2}\right)_{\ell}\right|^{2} \int_{0}^{1} \mathrm{~d} x \mathrm{~d} y \mathrm{~d} z\left[\ln \left(\frac{(1-y) m_{\ell}^{2}+y m_{\omega_{2}}^{2}-y(1-y) M_{\psi_{1}}^{2}-x z m_{Z}^{2}}{y m_{\ell}^{2}+(1-y) m_{\omega_{2}}^{2}-y(1-y) M_{\psi_{1}}^{2}-x z m_{Z}^{2}}\right)\right. \\
& \left.+\frac{x z m_{Z}^{2}-y^{2} M_{\psi_{1}}^{2}}{(1-y) m_{\ell}^{2}+y m_{\omega_{2}}^{2}-y(1-y) M_{\psi_{1}}^{2}-x z m_{Z}^{2}}\right]
\end{aligned}
$$

[1] P. Minkowski, Phys. Lett. 67B, 421 (1977); T. Yanagida, Conf. Proc. C7902131, 95 (1979); Prog. Theor. Phys. 64, 1103 (1980); M. Gell-Mann, P. Ramond, and R. Slansky, Conf. Proc. C C790927, 315 (1979); R. N. Mohapatra and G. Senjanovic, Phys. Rev. Lett. 44, 912 (1980).

[2] J. Schechter and J. W. F. Valle, Phys. Rev. D 22, 2227 (1980).

[3] W. Konetschny and W. Kummer, Phys. Lett. 70B, 433 (1977); R. N. Mohapatra and G. Senjanovic, Phys. Rev. Lett. 44, 912 (1980); M. Magg and C. Wetterich, Phys. Lett. 94B, 61 (1980); G. Lazarides, Q. Shafi, and C. Wetterich, Nucl. Phys. B181, 287 (1981).

[4] R. Foot, H. Lew, X. G. He, and G. C. Joshi, Z. Phys. C 44, 441 (1989).

[5] A. Zee, Phys. Lett. 93B, 389 (1980); 95B, 461(E) (1980).

[6] A. Zee, Nucl. Phys. B264, 99 (1986).

[7] K. S. Babu, Phys. Lett. B 203, 132 (1988).

[8] T. P. Cheng and L. F. Li, Phys. Rev. D 22, 2860 (1980).

[9] M. Gustafsson, J. M. No, and M. A. Rivera, Phys. Rev. Lett. 110, 211802 (2013); 112, 259902 (2014); Phys. Rev. D 90, 013012 (2014).

[10] L. M. Krauss, S. Nasri, and M. Trodden, Phys. Rev. D 67, 085002 (2003); A. Ahriche and S. Nasri, J. Cosmol. Astropart. Phys. 07 (2013) 035.

[11] M. Aoki, S. Kanemura, and O. Seto, Phys. Rev. Lett. 102, 051805 (2009); Phys. Rev. D 80, 033007 (2009); M. Aoki, S. Kanemura, and K. Yagyu, Phys. Rev. D 83, 075016 (2011).

[12] E. Ma, Phys. Rev. D 73, 077301 (2006); J. Kubo, E. Ma, and D. Suematsu, Phys. Lett. B 642, 18 (2006).

[13] V. A. Kuzmin, V. A. Rubakov, and M. E. Shaposhnikov, Phys. Lett. 155B, 36 (1985).

[14] S. Nasri and S. Moussa, Mod. Phys. Lett. A 17, 771 (2002); S. Kanemura, T. Nabeshima, and H. Sugiyama, Phys. Lett. B 703, 66 (2011).

[15] P. H. Gu and U. Sarkar, Phys. Rev. D 77, 105031 (2008).

[16] S. Kanemura, K. Sakurai, and H. Sugiyama, Phys. Rev. D 96, 095024 (2017).

[17] S. Kanemura and H. Sugiyama, Phys. Lett. B 753, 161 (2016).

[18] S. Kanemura, K. Sakurai, and H. Sugiyama, Phys. Lett. B 758, 465 (2016).

[19] G. Aad et al. (ATLAS Collaboration), J. High Energy Phys. 11 (2015) 211.

[20] V. Khachatryan et al. (CMS Collaboration), Phys. Lett. B 749, 337 (2015).
[21] CMS Collaboration, CERN Report No. CMS-PAS-HIG-16005, 2016.

[22] A. M. Sirunyan et al. (CMS Collaboration), J. High Energy Phys. 06 (2018) 001.

[23] M. Aoki, S. Kanemura, K. Sakurai, and H. Sugiyama, Phys. Lett. B 763, 352 (2016).

[24] B. Pontecorvo, Zh. Eksp. Teor. Fiz. 33, 549 (1957) [Sov. Phys. JETP 6, 429 (1957)]; Zh. Eksp. Teor. Fiz. 34, 247 (1957) [Sov. Phys. JETP 7, 172 (1958)]; Zh. Eksp. Teor. Fiz. 53, 1717 (1967) [Sov. Phys. JETP 26, 984 (1968)].

[25] Z. Maki, M. Nakagawa, and S. Sakata, Prog. Theor. Phys. 28, 870 (1962).

[26] M. Tanabashi et al. (Particle Data Group), Phys. Rev. D 98, 030001 (2018).

[27] A. M. Baldini et al. (MEG Collaboration), Eur. Phys. J. C 76, 434 (2016).

[28] B. Aubert et al. (BABAR Collaboration), Phys. Rev. Lett. 104, 021802 (2010).

[29] U. Bellgardt et al. (SINDRUM Collaboration), Nucl. Phys. B299, 1 (1988).

[30] K. Hayasaka et al., Phys. Lett. B 687, 139 (2010).

[31] V. Khachatryan et al. (CMS Collaboration), Phys. Lett. B 763, 472 (2016).

[32] M. Escudero, A. Berlin, D. Hooper, and M. X. Lin, J. Cosmol. Astropart. Phys. 12 (2016) 029.

[33] E. W. Kolb and M. S. Turner, Front. Phys. 69, 1 (1990).

[34] M. Aaboud et al. (ATLAS Collaboration), Eur. Phys. J. C 78, 995 (2018).

[35] A. Loureiro et al., arXiv:1811.02578.

[36] E. Kou et al. (Belle-II Collaboration), arXiv:1808.10567.

[37] L. Calibbi and G. Signorelli, Riv. Nuovo Cimento 41, 1 (2018).

[38] S. Banerjee, B. Bhattacherjee, M. Mitra, and M. Spannowsky, J. High Energy Phys. 07 (2016) 059.

[39] I. Chakraborty, A. Datta, and A. Kundu, J. Phys. G 43, 125001 (2016).

[40] N. Aghanim et al. (Planck Collaboration), arXiv: 1807.06209.

[41] J. Herrero-Garcia, E. Molinaro, and M. A. Schmidt, Eur. Phys. J. C 78, 471 (2018).

[42] E. Aprile et al. (XENON Collaboration), Phys. Rev. Lett. 119, 181301 (2017).

[43] X. Cui et al. (PandaX-II Collaboration), Phys. Rev. Lett. 119, 181302 (2017).

[44] D. S. Akerib et al. (LUX Collaboration), Phys. Rev. Lett. 118, 021303 (2017). 
[45] V. Khachatryan et al. (CMS Collaboration), J. High Energy Phys. 02 (2017) 135.

[46] S. Schael et al. (ALEPH, DELPHI, L3, OPAL, and SLD Collaborations, LEP Electroweak Working Group, SLD Electroweak Group, and SLD Heavy Flavour Group), Phys. Rep. 427, 257 (2006).

[47] S. Kanemura, T. Kasai, G. L. Lin, Y. Okada, J. J. Tseng, and C. P. Yuan, Phys. Rev. D 64, 053007 (2001).

[48] M. Aoki and S. Kanemura, Phys. Lett. B 689, 28 (2010).
[49] A. Ahriche, S. Nasri, and R. Soualah, Phys. Rev. D 89, 095010 (2014).

[50] M. Aoki, S. Kanemura, and O. Seto, arXiv:1008.2407.

[51] V. Rentala, W. Shepherd, and S. Su, Phys. Rev. D 84, 035004 (2011).

[52] M. Aoki, S. Kanemura, and K. Yagyu, Phys. Lett. B 702, 355 (2011); 706, 495(E) (2012).

[53] K. Enomoto, S. Kanemura, K. Sakurai, and H. Sugiyama (in preparation). 